UNIVERSITÀ CATTOLICA DEL SACRO CUORE

Dipartimento di Economia e Finanza

\author{
Working Paper Series
}

Citizen-Editors' Endogenous Information Acquisition and News Accuracy

Francesco Sobbrio

Working Paper n. 5

November 2013

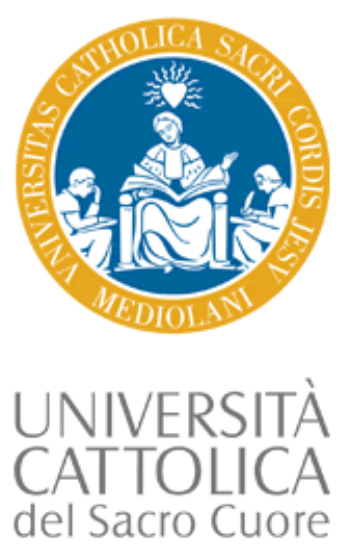




\title{
Citizen-Editors' Endogenous Information Acquisition and News Accuracy
}

\author{
Francesco Sobbrio \\ Università Cattolica del Sacro Cuore
}

Working Paper n. 5

November 2013

\author{
Dipartimento di Economia e Finanza \\ Università Cattolica del Sacro Cuore \\ Largo Gemelli 1 - 20123 Milano - Italy \\ tel: +39.02 .7234 .2976 - fax: +39.02 .7234 .2781$ \\ e-mail: dip.economiaefinanza@unicatt.it
}

The Working Paper Series promotes the circulation of research results produced by the members and affiliates of the Dipartimento di Economia e Finanza, with the aim of encouraging their dissemination and discussion. Results may be in a preliminary or advanced stage. The Dipartimento di Economia e Finanza is part of the Dipartimenti e Istituti di Scienze Economiche (DISCE) of the Università Cattolica del Sacro Cuore. 


\title{
Citizen-Editors' Endogenous Information Acquisition and News Accuracy*
}

\author{
Francesco Sobbrio ${ }^{\dagger}$
}

First version: June, 2009

This version: October, 2013

\begin{abstract}
This paper provides a model of the market for news where profit-maximizing media outlets choose their editors from a population of rational citizens. The analysis identifies a novel mechanism of media bias: the bias in a media outlet's news reports is the result of the slanted endogenous information acquisition strategy of its editor. In particular, the results show that the expected accuracy of news reports is lower the more ideological an editor is. Nevertheless, citizens find it optimal to acquire information from a media outlet whose editor has similar ideological preferences. Depending on the distribution of citizens' ideological preferences, a media outlet may choose an ideological editor even in a monopolistic market. Moreover, ideological editors are more likely to be present in the market for news: i) the higher the number of media outlets competing in the market for news; ii) the lower the opportunity cost that citizens have to incur to acquire information.
\end{abstract}

JEL Classification: D81, D83, L82

Key Words: Media Bias, Slant, Information Acquisition, Valence, Competition

*A previous version of the paper circulated under the title "A Citizen-Editors Model of News Media". I am very grateful to seminar participants at the Catholic University of Milan, Institut d'Anàlisi EconòmicaCSIC, Universitat de Barcelona, University of Bologna, Universitè catholique de Louvain, University of Namur, University of Padova; and at SAEe 2011, Congress of the European Economic Association, 7th Workshop in Media Economics, 2009 European Meeting of the Econometrics Society, the 2009 Meeting of the Association for Public Economic Theory, the 8th Journées Louis-André Gérard-Varet, the 2009 Meeting of the European Public Choice Society. The usual disclaimers apply.

†Catholic University of Milan. Email: francesco.sobbrio@unicatt.it 


\section{Introduction}

The importance of news media on the overall functioning of democracies is well documented by the extensive empirical evidence showing the significant influence of media on political outcomes. ${ }^{1}$ At the same time, journalists and communications scholars have provided substantial anecdotal evidence suggesting that the information supplied by news media to their viewers is often far from being "fair and balanced" (e.g., Goldberg, 2002; Alterman, 2003; Bagdikian, 2004; Davies, 2008). Significant deviations from the standard of unbiased news seem to be present even in fairly competitive media markets as, for example, the US. Indeed, a recent empirical literature in economics and political science has shown the presence of a systematic bias in the market for news using a variety of instruments to measure such bias (e.g., Groseclose and Milyo, 2005; Ho and Quinn, 2008; Gentzkow and Shapiro, 2010, Larcinese et al., 2011; Puglisi and Snyder, 2011). ${ }^{2}$ In parallel, a fast growing theoretical literature has provided (supply-driven or demand-driven) economic rationales for the presence of such systematic bias in the media by focusing on various incentives to bias the information supplied to media viewers (e.g., Mullainathan and Shleifer, 2005; Baron, 2006; Besley and Prat, 2006; Gentzkow and Shapiro, 2006; Annand et al., 2007; Chan and Suen, 2008; Ellman and Germano, 2009; Anderson and McLaren, 2012). ${ }^{3}$ Since these theoretical contributions take the information available to media outlets as exogenously given, they all assume, implicitly or explicitly, media outlets to bias their news reports by either selectively omitting a subset of their (exogenously given) information or by framing this information using an ideologically charged language.

Differently from the existing literature, this paper analyzes the endogenous acquisition of information by media editors and shows that the bias in media reports may arise from the way media editors gather information in the first place, rather than from the selective omission (or ideological framing) of exogenously given information. That is, the paper points out that the bias in a media outlet's news reports may be the result of the slanted optimal information acquisition strategy of its editor. In particular, the results show that a moderate editor (i.e., one who is ex-ante indifferent between a leftist or a rightist candidate) uses a balanced information acquisition strategy. The amount of evidence in support of the leftist candidate that she requires in order to stop collecting information and endorse such candidate is the same as the one she requires to endorse the rightist candidate. Instead, an ideological editor (i.e., one who, ex-ante, always prefers either the leftist or the rightist candidate) acquires information in a slanted way. A small amount of evidence in support of the leftist candidate is sufficient to induce a leftist editor to stop investing in information

\footnotetext{
${ }^{1}$ See, among the others, Strömberg, 2004a; Gentzkow, 2006; Eisensee and Strömberg, 2007; OberholzerGee and Waldfogel, 2009; Snyder and Strömberg, 2010; Chiang and Knight, 2011; Gentzkow et al. 2011; Drago et al. 2013.

${ }^{2}$ For evidence on the empirical effects of media bias see DellaVigna and Kaplan (2007), Gerber et al. (2009), DellaVigna and Gentzkow (2010) and Enikolopov et al. (2011).

${ }^{3}$ See Prat and Strömberg (2013) for an extensive survey of the literature on the political economy of mass media.
} 
acquisition and endorse that candidate. On the other hand, such an editor would endorse the rightist candidate only after having collected a large amount of evidence in support of that candidate.

The model analyses a market for news driven by the citizens' demand for information. Citizens have to choose between two alternative candidates (or policies). Citizens differ in their ideological preferences, but all equally value the valence (i.e., quality) of alternative candidates (or public benefit of alternative policies). Citizens may acquire some information about the quality of different candidates by watching news reports. News reports are produced by editors chosen by media outlets from the population of citizens. That is, once chosen by a media outlet, a citizen-editor can gather (costly) information about the candidates' quality and then report it to the viewers. Since citizen-editors with different ideological preferences have different optimal information acquisition strategies, a rational leftist (or rightist) citizen may prefer to watch the news reports supplied by a like-minded editor (i.e., an editor with similar ideological preferences) simply because the set of information acquired by such an editor provide her with a higher expected utility with respect to the one acquired by a moderate editor. Hence, while rational citizens always want any media editor to never omit any available information, they may still prefer a like-minded editor to a moderate one, due to the endogenous acquisition of costly information by citizen-editors. Media outlets anticipate this behavior by citizens and hence they choose their editors taking into account the expected demand for news reports produced by editors with different ideological preferences. That is, by choosing a more leftist, moderate or rightist editor, media outlets implicitly choose their product location in the political space.

Overall, by identifying a novel mechanism of media bias, the paper contributes to the understanding of both the supply-side and the demand-side of the market for news. In particular, for what concerns the supply-side of the market for news, the main insights of the model are as follows:

S.1 Editor's ideology and accuracy of news reports: the more ideological an editor is, the lower the expected accuracy of her news reports (i.e., the more extreme her ideological preferences, the lower the expected number of signals she collects and the higher the probability of her endorsing the low-valence candidate).

This result derives from the different optimal information acquisition strategies of editors with different ideological preferences. In particular, moderate editors are be the ones who (in expectation) collect the most signals before endorsing a candidate. Accordingly, they are also the ones with the lower expected probability of endorsing the low-valence candidate. Instead, the more ideological an editor is (i.e., the ideologically closer she is to one of the two candidates, ex-ante), the more likely that she ends up endorsing the ideologically-closer candidate. Moreover, the lower is the amount of evidence in favor of that candidate upon which she bases such an endorsement. 
This theoretical mechanism and results are consistent with different pieces of evidence. First, by surveying journalists in five different countries (i.e., USA, UK, Germany, Italy and Sweden), Patterson and Wolfgang (1996) show the presence of a significative positive correlation between a journalist's ideological preferences and their news decisions. Moreover, the model predictions regarding the higher accuracy of news by moderate editors, are in line the observed patterns of perceived accuracy and bias in news report in the US. For example, the following graph draws from survey evidence gathered by the Pew Research Center. In particular, the data suggests that the percentage of people who think that news media do not report accurate information is positively correlated with the percentage of people who think that news media are politically biased, in a given year. ${ }^{4}$

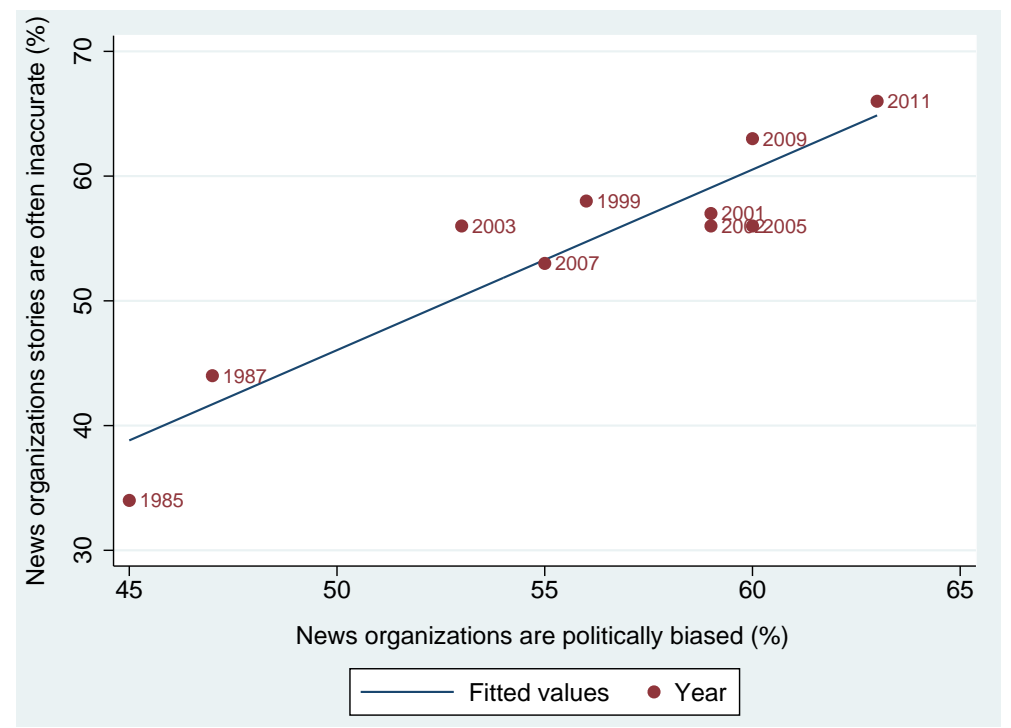

Fig. 1. Perceived accuracy and bias of US news organizations (Source: Pew Research Center, 2012)

S.2 Valence and news accuracy: the expected accuracy of news reports is higher on issues where the valence component is higher.

Since the valence component captures the importance of the "quality" of the issue/candidate with respect to the ideology, the model predicts that on issues where the ideological component is likely to be less relevant, editors are likely to deliver more accurate news reports. This is consistent with the evidence provided by Puglisi and Snyder (2011) who show that US newspapers provide more conservative endorsements on ballot propositions regarding economic issues with respect to the ones on social issues. As, arguably, the ideological component is higher on social issues with respect to economic issues, the model would predict that journalists are more likely to acquire less information before making an endorsement (and thus are more likely to make endorsement in line with their ex-ante ranking over alternatives). Moreover, this result is also consistent with the fact that the news media perceived as most accurate in the US are local TV news (Pew Research Center, 2012). As citizens are

\footnotetext{
${ }^{4}$ Appendix B provides further evidence on this issue.
} 
likely to care relatively more about the valence component than the ideological one when it comes to local issues, this evidence is in line with the results of the model.

S.3 Media slant and competition: ideological editors are more likely to be present in the market for news the higher the number of media outlets competing in the market for news.

This result is typical in models of demand-driven media bias (e.g., Mullainathan and Shleifer, 2005; Annand et al., 2007; Chan and Suen, 2008). It follows from the incentives of profit-maximizing media outlets to differentiate their news products. In particular, the analysis shows that, even in the case where citizens are uniformly distributed in the policy space, there is a threshold in the number of media outlets present in the market for news above which media outlets may find it optimal to choose ideological editors. As a consequence, markets for news characterized by a higher degree of competition are likely to have a higher degree of polarization of news media. This result is consistent with the different degrees of ideological polarization of news sources observed in online and offline media markets (e.g., online newspapers and blogs with respect to traditional newspapers and TV). As argued by Sunstein (2007), the dramatic expansion in online media outlets seems to have increased the degree of polarization in the market for news. Indeed, Gentzkow and Shapiro (2011) show that the "most extreme Internet sites are far more polarized than any source offline" (Gentzkow and Shapiro, 2011, page 15). ${ }^{5}$ Moreover, the above result is also in line with historical evidence on US newspapers showing that economic competition enhances ideological diversity (Gentzkow et al., 2012).

S.4 Media slant and opportunity cost of acquiring information: ideological editors are more likely to be present in the market for news the lower the opportunity cost that citizens have to incur to acquire information.

This result is driven by the demand for news coming from "extremist" citizens. When the opportunity cost of acquiring information is high, the expected benefit of watching news reports for "extremist" citizens is lower than the cost. Hence, in this case, media outlets are likely to choose moderate editors since the bulk of the demand for news comes from moderate citizens. Instead, when the opportunity cost is low, even "extremist" citizens may find convenient to watch news reports when such news reports come from an editor with similar idiosyncratic preferences. Hence, a media outlet may find it optimal to choose an ideological editor to capture this demand for news by ideological citizens. A clear application of such a result is represented by the market for news in the broadcast media sector with respect to the press. The opportunity cost of watching a broadcast media report is arguably

\footnotetext{
${ }^{5}$ While both Sunstein (2007) and Gentzkow and Shapiro (2011) point out the higher degree of polarization of online media sources with respect to the offline ones, Gentzkow and Shapiro (2011) show that the polarization of online media viewers may not necessarily be significatively higher than the one of viewers in offline media markets.
} 
lower than the one of reading a newspaper. The analysis thus suggests that, ceteris paribus, the share of moderate editors present in the press sector should be higher than the one of the broadcast media sector. Moreover, "extremist" citizens should be more likely to acquire information from broadcast media than from newspapers and broadcast media should face a higher overall demand with respect to the one faced by the press.

At the same time, the model provides the following insights on the demand-side of the market for news:

\section{D.1 Demand for slanted news: rational citizens find it optimal to acquire information from} a liked-minded source of news.

An economic rational for the demand for liked-minded news is already present in other models of demand-driven media bias (Mullainathan and Shleifer, 2005; Gentzkow and Shapiro, 2006; Annand et al., 2007; Chan and Suen, 2008). In the context of the present paper, this result derives from the fact that, when choosing among different media outlets, rational citizens anticipate that the news reports coming from media editors with different ideological preferences will be different simply because the information acquired by each of these editors are different. Indeed, as in the literature on citizen-candidates voters know that a candidate can only credibly commit to her preferred policy (Osborne and Slivinsky, 1996; Besley and Coate, 1997), in the present paper viewers know that a media outlet's editor can only credibly commit to her own optimal information acquisition strategy. Accordingly, rational (non-moderate) citizens prefer a media outlet with a like-minded editor simply because they derive a higher utility from the set of information acquired by such an editor with respect to the one acquired by a moderate editor. ${ }^{6}$

The results are consistent with the empirical results of Gentzkow and Shapiro (2010). Using zip-code level data on newspaper circulation in the US, they show that the demand for right-wing newspaper is higher in markets with a higher proportion of Republicans. Moreover, they find that ownership has little or no role in media slant. ${ }^{7}$ Similarly, Puglisi and Snyder (2011) find that, on average, the ideological location of US newspapers corresponds to the one of the median voter in their states. Finally, Gentzkow et al. (2012) provide evidence on the US newspapers in the early 20th century suggesting that consumers have strong preferences for like-minded sources of news. ${ }^{8}$ My results suggest that such findings may be the result of the demand for costly information by rational individuals and the consequent optimal ideological location of news by profit maximizing media outlets. ${ }^{9}$

\footnotetext{
${ }^{6}$ At the same time, since the more extreme the idiosyncratic preferences of an editor are, the lower the accuracy of its news reports, there is always an upper bound on the possible "extremism" of an editor above which the demand for news of citizens is strictly decreasing.

${ }^{7}$ Specifically, they find that "the slant of co-owned papers is only weakly (and statistically insignificantly) correlated to a newspaper's political alignment" (Gentzkow and Shapiro, 2010, page 38).

${ }^{8}$ In particular, they show that "an increase of 10 percentage points in the proportion of a town's votes going to Republicans increases the relative circulation of Republican papers in the town by 10 percent" (Gentzkow et al. 2012, page 3).

${ }^{9}$ Calvert (1985) was the first to point out the positive value of a biased source of information for a rational
} 
D.2 Editor's ideology and citizen's voting behavior:only an endorsement by a like-minded editor in favor of the ideologically-farther candidate would change the ex-ante ranking of preferences over candidates of a leftist or rightist citizen.

As discussed above, a leftist citizen knows that a leftist editor would endorse the rightist candidate only after having acquired a large amount of evidence in favor of that candidate. Instead, a report in favor of the rightist candidate by a moderate or rightist editor would not contain enough evidence to convince a leftist citizen to choose such a candidate. This theoretical mechanism is in line with the empirical analysis of newspaper endorsements and media influence in the US by Chiang and Knight (2011). Indeed, consistently with the predictions of my model, Chiang and Knight find that the degree of influence of a newspaper on voters depends on the credibility of the endorsement. ${ }^{10}$

The paper is organized as follows. Section 2 describes the model and the structure of the game. Section 3 derives the optimal information acquisition strategy by citizen-editors and discusses its implications for the accuracy of news reports. Section 4 discusses the demand for news. Section 5 contains the results on the optimal choice of editors by media outlets. Section 6 discusses the rationale for acquiring information and the implications of the results for citizen's welfare. Section 7 concludes. Appendix A discusses the robustness of the results. Appendix B presents some additional empirical evidence on the correlation between media bias and news accuracy. All the proofs are provided in Appendix C.

\section{The Model}

\subsection{Structure of the game}

There are two alternative candidates/policies $L$ and $R$ where $L=0$ and $R=1$, i.e., the policy space is $\{0 ; 1\}$. A continuum of citizens of measure one have to decide which candidate $P \in\{L ; R\}$ to choose. There are two possible states of the world $s \in\{l, r\}$. To preserve symmetry, the common prior belief that the state of the world is $r$ is assumed to be $\operatorname{Pr}(s=$ $r)=1 / 2$. Citizens care about the ideological distance between their idiosyncratic preferences and the candidates' policy platforms (i.e., euclidean distance between their preferences and the ones of the chosen candidate). At the same time, citizens also care about the valence (i.e., quality) of the candidates. The valence component is captured by an additive constant in the citizen's utility function. That is, regardless of her idiosyncratic policy preferences, each citizen gets an extra positive payoff when she chooses the high-valence candidate and a

\footnotetext{
decision-maker. See also Cukierman and Tommasi (1998) and Li and Suen (2004).

${ }^{10}$ Specifically, Chiang and Knight show that "endorsements for the Democratic candidate from left-leaning newspapers are less influential than are endorsements from neutral or right-leaning newspapers and likewise for endorsements for the Republican candidate" (Chiang and Knight, 2011, page 817).
} 
negative one when the low-valence candidate is chosen. ${ }^{11}$ Hence, citizen $i$ 's utility function is:

$$
u_{i}\left(P, x_{i}\right)=\delta I_{s} I_{p}-\left|P-x_{i}\right|
$$

where $x_{i}$ represents the idiosyncratic (i.e., ideological) policy preference of citizen $i$ and $\delta$ represents the valence parameter. Moreover, without loss of generality $\delta \in\left(0, \frac{1}{2}\right]$ and:

$$
I_{s}=\left\{\begin{array}{r}
1 \text { if } s=l \\
-1 \text { if } s=r
\end{array} \quad \text { and } \quad I_{p}=\left\{\begin{array}{r}
1 \text { if } P=L \\
-1 \text { if } P=R
\end{array}\right.\right.
$$

As a consequence, candidate $L$ gives a higher utility to citizens when the state of the world is $l$ than when the state is $r$ (viceversa for candidate $R$ ). ${ }^{12}$ In other words, $L$ and $R$ represent the alternative political platforms of the two candidates and $2 \delta$ represents the difference in the valence of the two candidates in each state of the world. ${ }^{13}$ The idiosyncratic preferences of citizens are distributed with a common knowledge c.d.f. $F(x)$ with density function $f(x)$ where $\operatorname{supp}[f(x)]=[0,1]$. To avoid the presence of exogenous asymmetries, the analysis focuses on distributions that are symmetric and monotone in the sub-intervals $x \in\left[0, \frac{1}{2}\right]$ and $x \in\left[\frac{1}{2}, 1\right] .{ }^{14}$ For the sake of clarity of exposition, two different cases will be considered regarding the shape of the density function. The first case applies when the density of citizens' idiosyncratic preferences is increasing moving away from the "extremes" (i.e., towards $1 / 2$ ). That is:

$$
\frac{\partial f(x)}{\partial x}\left\{\begin{array}{l}
\geq 0 \text { for } x \leq \frac{1}{2} \\
\leq 0 \text { for } x>\frac{1}{2}
\end{array}\right.
$$

The second case applies when the density of citizens' idiosyncratic preferences is increasing moving away from the median citizen (i.e., towards 0 and 1). That is:

$$
\frac{\partial f(x)}{\partial x}\left\{\begin{array}{l}
<0 \text { for } x<\frac{1}{2} \\
>0 \text { for } x \geq \frac{1}{2}
\end{array}\right.
$$

(Condition B)

The state contingent utilities of citizen $i$ are, thus, as follows:

$$
u_{i}(L \mid s)=\left\{\begin{aligned}
\delta-x_{i} & \text { if } s=l \\
-\delta-x_{i} & \text { if } s=r
\end{aligned} \quad \text { and } \quad u_{i}(R \mid s)=\left\{\begin{aligned}
-\delta+x_{i}-1 & \text { if } s=l \\
\delta+x_{i}-1 & \text { if } s=r
\end{aligned}\right.\right.
$$

\footnotetext{
${ }^{11}$ As usual in the literature on the demand for news (e.g., Strömberg, 2004b; Mullainathan and Shleifer, 2005; Baron, 2006; Gentzkow and Shapiro, 2006; Chan and Suen, 2008; Anderson and McLaren, 2012) it is assumed that citizens receive utility from choosing a given candidate/alternative per se. Section 6.1 provides a discussion on this assumption.

${ }^{12}$ For a similar specification of the voters' utility function see, for example, Aragones and Palfrey (2002).

${ }^{13} \mathrm{As}$ an alternative interpretation of the model, $L$ and $R$ can be seen as two alternative policies (e.g. implementing Kyoto's protocol or not). Hence, if the state of the world is $l$ then the public benefits/cost ratio of policy $L$ is higher than the one of $R$ (viceversa if $s=r$ ). That is, if the state of the world is $l$ policy $L$ is the most efficient one.

${ }^{14}$ For example, the families of Uniform, Normal, and Cauchy distribution functions satisfy such property.
} 
Before deciding which candidate to choose, each citizen may access a media outlet's news report by paying an opportunity $\operatorname{cost} C$. Specifically, the media industry is composed by $K \geq 1$ media outlets. Each media outlet is assumed to be maximizing its viewership in order to maximize its advertising revenues. In order to produce news reports, each media outlet has to choose an editor from the population of citizens. Once chosen, a citizen-editor is endowed with a (costly) technology that allows her to collect evidence on the state of the world. Specifically, a citizen-editor has to incur a cost $c$ any time she decides to draw a signal on the state of the world (e.g., effort she has to exert to acquire information, opportunity cost of sending reporters to investigate an issue, etc.). ${ }^{15}$ Indeed, media editors (or, more generally, journalists) are the ones spending time and exerting effort on a daily-basis to acquire information to produce news reports. That is, media outlets/owners do not directly bear this day to day cost of information acquisition. ${ }^{16}$

The signal space is given by $\Sigma=\left\{\sigma_{l}, \sigma_{r}\right\}$ and the signal likelihood function is as follows:

$$
\operatorname{Pr}\left(\sigma_{l} \mid s=l\right)=\operatorname{Pr}\left(\sigma_{r} \mid s=r\right)=\theta
$$

where $\theta \in\left(\frac{1}{2}, 1\right)$ represents the precision of the signal. Hence, $\sigma_{l}\left(\sigma_{r}\right)$ represents a signal providing evidence in support of candidate $L(R)$ being the high-valence candidate. Essentially, as discussed extensively in the next section, the citizen-editor has to decide how many $\sigma_{l}$ and $\sigma_{r}$ signals she has to collect before producing a news report. Then, if a citizen decides to pay the opportunity $\operatorname{cost} C$ of accessing a media outlet's report she will update her beliefs using Bayes' rule. Hence, the demand for news reports that a media outlet faces is a function of the type of editor that it has chosen. That is, given an editor with idiosyncratic preferences $x_{e}$, the profit function of media outlet $k$ is $\Pi_{k}\left(x_{e}\right)=D_{k}\left(x_{e}\right)$, where $D_{k}\left(x_{e}\right)$ is the demand for the news report produced by the media outlet. ${ }^{17}$ To summarize, the timing of the game is as follows:

\begin{tabular}{|c|c|c|c|c|}
\hline Nature draws & Media outlets choose & Each editor samples & Citizens decide whether & Citizens choose their \\
\hline state of the & their editors from the & and then produces a & to watch a media outlet's & preferred candidate. \\
\hline world $l$ or $r$ & population of citizens & news report & $\begin{array}{l}\text { report and if so, update } \\
\text { their beliefs. }\end{array}$ & Payoffs are realized \\
\hline
\end{tabular}

Fig. 2. Timing of the Game

\footnotetext{
${ }^{15}$ By "editor" I refer to what is usually called "Editor-in-Chief" for a newspaper and "Managing Editor" in the broadcast media sector. More in general, the model applies to the choice of a profit maximizing media outlet regarding the type of journalists to be hired.

${ }^{16}$ Moreover, as discussed extensively in Section A.1 in Appendix A, since media owners cannot monitor/observe the signals acquired by media editors, they cannot choose the number of signals gathered by a media editor.

${ }^{17}$ See section A.1 in Appendix A for a discussion on the structure of media outlets' profits.
} 


\subsection{News reports and citizens' preferences over candidates}

Before turning to the analysis of the optimal information acquisition by citizen-editors and the optimal choice of editors by profit-maximizing media outlets, I provide here a brief discussion of the citizens' preferences over candidates contingent on the news report observed.

Suppose that a citizen receives a news report containing $n_{l}$ signals $\sigma_{l}$ and $n_{r}$ signals $\sigma_{r}$ on the state of the world. Then, the citizen's posterior beliefs are:

$$
\operatorname{Pr}\left(s=r \mid n_{l}, n_{r}\right)=\frac{\theta^{n_{r}-n_{l}}}{\theta^{n_{r}-n_{l}}+(1-\theta)^{n_{r}-n_{l}}}
$$

Therefore, denoting $n=n_{r}-n_{l}$, the citizen's posterior beliefs may be summarized as follows:

$$
\mu(n)=\frac{1}{1+\left(\frac{1-\theta}{\theta}\right)^{n}}
$$

Hence, a citizen with idiosyncratic preferences $x_{i}$ would prefer candidate $R$ to candidate $L$ whenever:

$$
\mu(n)>\frac{1}{4 \delta}\left(2 \delta-2 x_{i}+1\right)=\mu\left(\hat{n}_{i}\right)=\hat{\mu}_{i}
$$

That is $\hat{n}_{i}$ is the difference in the number of signals in favor of state $r$ which makes citizen $i$ being indifferent between candidates $R$ and $L$. Notice that for $\delta=\frac{1}{2}$, then $\hat{\mu}_{i} \geq 0, \forall i$. Hence, for $\delta=\frac{1}{2}$ all citizens would prefer candidate $L$ when $s=l$ and candidate $R$ when $s=r$. That is, when $\delta=\frac{1}{2}$, ex-post all citizens have the same ranking of preferences over candidates. Instead, for $0<\delta<\frac{1}{2}$ there will be some "stubborn" citizens who will always vote for the same candidate regardless of the state of the world. Moreover:

$$
\frac{\partial u_{i}(R \mid \mu(n))}{\partial \mu(n)}=-\frac{\partial u_{i}(L \mid \mu(n))}{\partial \mu(n)}=2 \delta, \forall i
$$

hence, the utility functions of citizens $i$ and $j$ are always parallel. For any exogenously given $\mu(n) \in(0,1)$, different citizens may have different ranking of preferences regarding candidates $L$ and $R$. Specifically:

$$
\hat{\mu}_{\frac{1}{2}}=\frac{1}{2} \quad \text { and } \quad \frac{\partial \hat{\mu}_{i}}{\partial x_{i}}<0
$$

Thus, citizens with more "rightist" preferences require less evidence in favor of $R$ in order to choose that candidate with respect to moderate citizens. Moreover, when a citizen cares more about the true state of the world (i.e., when the valence component is larger), her indifference threshold is closer to the one of a moderate citizen:

$$
\frac{\partial \hat{\mu}_{i}}{\partial \delta}=\frac{\left(2 x_{i}-1\right)}{4 \delta^{2}}\left\{\begin{array}{lll}
<0 & \text { if } & x_{i}<\frac{1}{2} \\
>0 & \text { if } & x_{i}>\frac{1}{2}
\end{array}\right.
$$

Hence, the more citizens care about the quality of different candidates, the less evidence in 
favor of the ideologically-farther candidate they require in order to vote for her.

\subsection{Information Acquisition by Citizen-Editors}

An editor faces a trade-off between the cost of acquiring a signal and the utility she gets from the informative content of each signal. ${ }^{18}$ Let $x_{e}$ represent the idiosyncratic preferences of a citizen-editor chosen by a media outlet. Denote $\tau_{e, m}(n)$ the decision of such a citizen-editor given that she has already drawn $m=\{0,1, \ldots . \infty\}$ signals and given a current difference of signals in favor of $r$ equal to $n$. Given any $m$ and $n$, the choice set of citizen-editor $e$ is $\Gamma_{m}(n)=\{L, R, d\}$. Thus she can choose candidate $L$ or $R$ or she can pay $c$ and draw another signal on the state of the world (i.e., choose $\tau_{e, m}(n)=d$, where $d$ stands for "draw"). Hence, the citizen-editor's problem is to find an optimal stopping rule. Specifically, the value function that editor $e$ maximizes after $m$ draws, given a current difference of signals in favor of state $r$ equal to $n$, is the following:

$$
V_{e}(n)=\left\{\begin{array}{l}
\max \left\{\begin{array}{l}
\delta(1-2 \mu(n))-x_{e} ; \\
\nu(n) V_{e}(n+1)+(1-\nu(n)) V_{e}(n-1)-c
\end{array}\right\} \text { if } \mu(n)<\hat{\mu}_{e} \\
\max \left\{\begin{array}{l}
\delta(2 \mu(n)-1)-\left(1-x_{e}\right) ; \\
\nu(n) V_{e}(n+1)+(1-\nu(n)) V_{e}(n-1)-c
\end{array}\right\} \text { if } \mu(n) \geq \hat{\mu}_{e}
\end{array}\right.
$$

where $\nu(n)=\mu(n) \theta+(1-\mu(n))(1-\theta)$. In other words, if after $m$ draws editor $e$ has a posterior $\mu(n)<\hat{\mu}_{e}$ she will decide either to stop acquiring signals and choose candidate $L$ with an expected payoff of $(1-\mu(n))\left(\delta-x_{e}\right)+\mu(n)\left(-\delta-x_{e}\right)$ or paying $c$ and getting another signal. In this case, with probability $\nu$ the editor will get signal $\sigma_{r}$ in which case the value function becomes $V_{e}(n+1)$ and with probability $(1-\nu)$ she will get signal $\sigma_{l}$ in which case the value function becomes $V_{e}(n-1)$. Instead, if after $m$ draws editor $e$ has a posterior $\mu(n) \geq \hat{\mu}_{e}$ she will decide either to stop acquiring information and choose candidate $R$ with an expected payoff of $(1-\mu(n))\left(x_{e}-\delta-1\right)+\mu(n)\left(x_{e}+\delta-1\right)$ or paying $c$ and getting another signal. In this case, with probability $\nu$ the editor will get signal $\sigma_{r}$ in which case the value function becomes $V_{e}(n+1)$ and with probability $(1-\nu)$ she will get signal $\sigma_{l}$ in which case the value function becomes $V_{e}(n-1) .{ }^{19}$

For clarity of exposition, in what follows I will refer to citizens and editors with idiosyncratic preferences $x_{i}=1 / 2$ as moderate citizens/editors. Hence, a citizen/editor is labeled as moderate if she only cares about the valence of the candidates (i.e., ex-ante she is indifferent between the two candidates). Instead, I will refer to citizens and editors with idiosyncratic preferences $x_{e} \neq 1 / 2$ as ideological citizens/editors. Hence, a citizen/editor

\footnotetext{
${ }^{18}$ Section A.1 in Appendix A provides a discussion on the robustness of the optimal information acquisition strategy by citizen-editors to the presence of incentive mechanisms.

${ }^{19}$ Notice that the value function of editor $e$ does not depend on how many draws she has already done (i.e., $m$ ), since the only relevant variable for her decision is the current difference of signals in favor of $r$ (i.e., the state variable is $n$ ).
} 
is labeled as ideological if, ex-ante, she always prefers one of the two candidates. Finally, a citizen/editor $i$ is labeled as more ideological than $j$ if her idiosyncratic preferences are closer to either 0 or 1 with respect to the idiosyncratic preferences of $j$.

\section{Editor's ideological preferences and news accuracy}

This section characterizes the properties of the optimal strategy of a citizen-editor (i.e., her optimal sampling strategy) as a function of the parameters of the model and of the editor's ideological preferences. The following proposition summarizes the results.

Proposition 1 Let $\underline{n}_{e}^{*}$ and $\bar{n}_{e}^{*}$ denote the thresholds in the number of signals in favor of $R$ such that a citizen-editor with ideological preferences $x_{e}$ stops acquiring information and endorses $R$ for $n \geq \bar{n}_{e}^{*}$ and stops acquiring information and endorses $L$ for $n \leq \underline{n}_{e}^{*}$. Then:

1. $\frac{d \underline{n}_{e}^{*}}{d x_{e}}<0, \frac{d \underline{n}_{e}^{*}}{d \delta}<0$ and $\frac{d \underline{n}_{e}^{*}}{d c}>0$

2. $\frac{d \bar{n}_{e}^{*}}{d x_{e}}<0, \frac{d \bar{n}_{e}^{*}}{d \delta}>0$ and $\frac{d \bar{n}_{e}^{*}}{d c}<0$

Moreover, a more ideological an editor requires even less signals in favor of the ideologicallycloser candidate than more in favor of the ideologically-further one in order to stop acquiring information and endorse that candidate.

The following graph illustrates the optimal strategy of editor $e$ after $m$ draws, given a current difference of signals in favor of $r$ equal to $n$ :

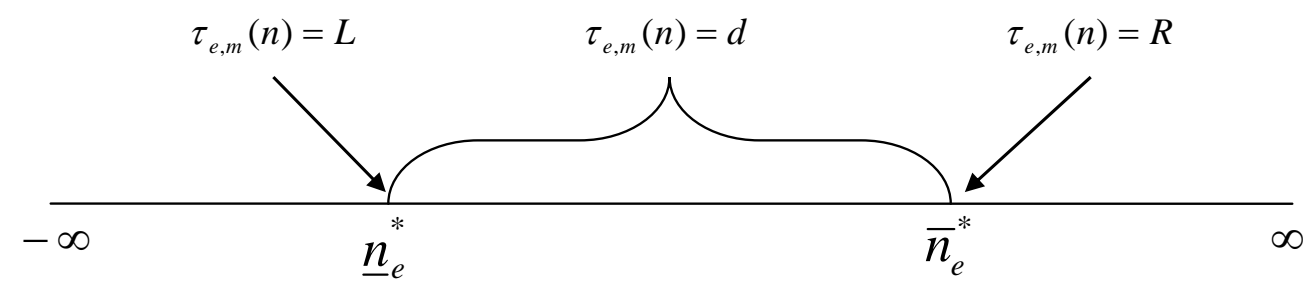

Fig. 3. Optimal Strategy of editor $e$

In other words, $\underline{n}_{e}^{*}$ is the threshold below which editor $e$ does not sample anymore and reports $\left|\underline{n}_{e}^{*}\right|$ more signals in favor of candidate $L$. Similarly, $\bar{n}_{e}^{*}$ is the threshold above which editor $e$ does not sample anymore and reports $\bar{n}_{e}^{*}$ more signals in favor of candidate $R$.

For any given $n$ a more "rightist" editor is always more likely to produce a report in favor of candidate $R$ than in favor of $L$, with respect to a more "leftist" editor. That is, $x_{e^{\prime}}>x_{e}$ implies that $\underline{n}_{e^{\prime}}^{*}<\underline{n}_{e}^{*}$ and $\bar{n}_{e^{\prime}}^{*}<\bar{n}_{e}^{*}$. Moreover, given editors $e$ and $e^{\prime}$ with $x_{e^{\prime}}<x_{e} \leq \frac{1}{2}$, then $\bar{n}_{e^{\prime}}^{*}-\underline{n}_{e^{\prime}}^{*}<\bar{n}_{e}^{*}-\underline{n}_{e}^{*}$. Hence, a more leftist editor requires even less signal in favor of $L$ than more in favor of $R$ to stop sampling, with respect to a more moderate editor. Similarly, given editors $e$ and $e^{\prime}$ with $x_{e^{\prime}}>x_{e} \geq \frac{1}{2}$, then $\bar{n}_{e^{\prime}}^{*}-\underline{n}_{e^{\prime}}^{*}<\bar{n}_{e}^{*}-\underline{n}_{e}^{*}$. Hence, a more rightist editor requires even less signals in favor of $R$ than more in favor of $L$ with respect to a more moderate editor. Therefore, the more moderate an editor is, the larger is her "information 
acquisition set" $N_{e}=\left\{n \mid \tau_{e, m}(n)=d\right\}$ (i.e., the set of the difference in the number of signals in favor of $r$ (or in favor of $l$ ) such that editor $e$ will keep sampling). ${ }^{20}$ At the same time, an increase in the importance of the valence component of the editor's utility function $(\delta)$ makes an editor sample more in both directions (i.e., $N_{e}$ becomes larger). Moreover, an increase in $\delta$ induces a leftist editor to increase her "leftist" stopping rule more than her "rightist" stopping rule (i.e., $\left|\underline{n}_{e}^{*}\right|$ increases more than $\bar{n}_{e}^{*}$ ). The opposite is true for a rightist editor. A higher $\delta$ is associated with more sampling in both directions and more symmetric stopping rules for all types of editors. Therefore, Proposition 1 suggests that when $\delta$ is higher any type of editor: i) acquires more information; ii) behaves as if she were more moderate (i.e., has more symmetric stopping rules).

Notice that, for $x_{e}=\frac{1}{2}, \bar{n}_{e}^{*}-\hat{n}_{e}=\hat{n}_{e}-\underline{n}_{e}^{*}$ and thus $\mu\left(\bar{n}_{e}^{*}\right)=1-\mu\left(\underline{n}_{e}^{*}\right)$. Moreover for $x_{e^{\prime}}>x_{e}:$

$$
\mu\left(\underline{n}_{e^{\prime}}^{*}\right)<\mu\left(\underline{n}_{e}^{*}\right)<1 / 2<\mu\left(\bar{n}_{e^{\prime}}^{*}\right)<\mu\left(\bar{n}_{e}^{*}\right)
$$

The above proposition has a corollary which provides one of the main insight of the paper regarding the link between the media editor's ideological preferences and the accuracy of her news reports.

Corollary 1 The expected number of signals acquired by an editor before endorsing a candidate and the expected probability of an editor endorsing the high-valence candidate $P$ are both:

i) Increasing the more moderate an editor is.

ii) Decreasing in the cost $c$ of gathering information and increasing in the valence parameter $\delta$.

This Corollary provides one of the key results of the paper. It shows that moderate editors are the ones providing the most accurate news reports (in expectation). Indeed, moderate editors are the ones acquiring the highest number of signals before stop sampling and endorsing one of the two candidates. Instead, the more ideological an editor is, the lower (in expectation) the amount of information that she would collect before making a news report. At the same time, more ideological editors are (ex-ante) more likely to endorse the low-valence candidate. Accordingly, the probability of choosing the low-valence candidate is decreasing in the "ideological distance" between an editor and the candidate. That is, more "rightist" editors are less likely to choose candidate $L$ when the high quality one is $R$. Instead, they are more likely to choose candidate $R$ when the high quality one is $L$. More generally, from an ex-ante perspective, moderate editors are less likely to make a report in favor of the low-quality candidate. This is due to the fact that the more moderate an editor is, the more symmetric her sampling strategy is and also the more information she acquires

\footnotetext{
${ }^{20}$ Notice that it is always the case that either $N_{e} \equiv \varnothing$ or $N_{e} \equiv\left\{\underline{n}_{e}^{*}, \underline{n}_{e}^{*}+1, \ldots \ldots, \bar{n}_{e}^{*}-1, \bar{n}_{e}^{*}\right\} \supseteq\{0\}$.
} 
before making a decision. Therefore, by taking on average a "more informed" decision, moderate editors are less likely to choose the low-quality candidate. Overall, this result points out the presence of a negative relation between the extremism of the ideological preferences of media editors (or, more generally, journalists) and the accuracy of news reports. ${ }^{21}$ Hence, the above Corollary sheds light on an issue which has not been addressed in the literature and that represents an additional element to enhance the understanding of the market for news. In particular, the evidence provided by Figure 1 in the introduction, as well as the one present in Figures B1 and B2 in Appendix B, is consistent with the theoretical predictions of Corollary 1.

Finally, as expected, when the cost of sampling is higher, editors will make more "errors" in the sense that they would be less likely to choose the high-valence candidate and would collect less signals before making a news report. Instead, when editors care more about the quality of candidates the expected number of signals collected increases and the probability of choosing the low-valence candidate decreases (since as shown by Proposition 1, when $\delta$ is higher editors acquire more information). This result is also consistent with the evidence provided by a recent report of the Pew Research Center showing that Local TV news are the most trusted sources of information. ${ }^{22}$ Indeed, arguably, local issues carry a lower degree of ideology and thus the above result suggests that on issues where the ideological component is less relevant, media editors are more likely to produce accurate news reports.

\section{The Demand for News}

This section analyzes the demand by citizens for the news reports of a media outlet as a function of the optimal stopping rules of its editor. Given the idiosyncratic preferences of a media outlet's editor, each citizen $i$ can infer the set of possible reports of a media outlet (i.e., citizen $i$ knows that the editor will either stop acquiring information after having collected $\underline{n}_{e}^{*}$ signals in favor of $L$ or $\bar{n}_{e}^{*}$ in favor of $R$ ). Hence, analogously to the literature on citizen-candidates where citizens know that a candidate has a personal commitment to implement a given policy, in the model citizens know that an editor has a personal commitment to implement a given information acquisition strategy. ${ }^{23}$ Notice that what really matters for citizen when deciding whether to access a media outlet's report is not the identity of the editor per se but rather her ideological preferences. As greatly exemplified by Chan and Suen (2008), news media have a clear incentive to make such ideological

\footnotetext{
${ }^{21}$ Notice that moderate editors do not have any higher intrinsic value per se. Moderate editors simply provide a useful benchmark since their perfectly symmetric stopping thresholds correspond to what is usually considered as a "fair and balanced" news report. For example, the idea of "fair and balanced" news reports was at the foundation of the FCC Fairness Doctrine in the US. Similarly, as stated by the BBC in the UK, "Impartiality lies at the heart of public service and is the core of the BBC's commitment to its audiences" (www.bbc.co.uk/guidelines/editorialguidelines). Nevertheless, as shown in the next section, these "fair and balanced" news reports are not necessarily the optimal ones from the perspective of every single citizen.

22 "Further Decline in Credibility Ratings for Most News Organizations" (PEW, 2012)

${ }^{23}$ See sections A.1 and A.2 in Appendix A for a discussion on this issue.
} 
preferences common knowledge. Indeed, "since news consumers prefer news from sources that share their ideological preferences, it is in the interest of a newspaper to establish a clear ideological reputation by hiring an editor with known views." Chan and Suen (2008, page 5$).{ }^{24}$

Let the citizens' action space be $A=\{W, N W\}$ where $W$ stands for watching the news reports and $N W$ for not watching the news reports. Then, the expected utility of citizen $i$ from not getting any news report from the media outlet is:

$$
U_{i}(N W)=\left\{\begin{array}{l}
U_{i}\left(L \mid \frac{1}{2}\right) \text { for } x_{i}<\frac{1}{2} \\
U_{i}\left(R \mid \frac{1}{2}\right) \text { for } x_{i}>\frac{1}{2}
\end{array}\right.
$$

If instead citizen $i$ decides to pay a cost $C$ to access the news report of an editor with idiosyncratic preferences $x_{e}$, her expected utility will be:

$$
\begin{aligned}
U_{i}\left(W, x_{e}\right)= & \operatorname{Pr}\left(n=\underline{n}_{e}^{*}\right) \max \left\{U_{i}\left(L \mid \mu\left(\underline{n}_{e}^{*}\right)\right) ; U_{i}\left(R \mid \mu\left(\underline{n}_{e}^{*}\right)\right)\right\} \\
& +\operatorname{Pr}\left(n=\bar{n}_{e}^{*}\right) \max \left\{U_{i}\left(L \mid \mu\left(\bar{n}_{e}^{*}\right)\right) ; U_{i}\left(R \mid \mu\left(\bar{n}_{e}^{*}\right)\right)\right\}-C
\end{aligned}
$$

Where the probabilities of reaching the two stopping threshold $\underline{n}_{e}^{*}$ and $\bar{n}_{e}^{*}$ are: ${ }^{25}$

$$
\operatorname{Pr}\left(n=\underline{n}_{e}^{*}\right)=\frac{2 \mu\left(\bar{n}_{e}^{*}\right)-1}{2\left[\mu\left(\bar{n}_{e}^{*}\right)-\mu\left(\underline{n}_{e}^{*}\right)\right]}
$$

and

$$
\operatorname{Pr}\left(n=\bar{n}_{e}^{*}\right)=\frac{1-2 \mu\left(\underline{n}_{e}^{*}\right)}{2\left[\mu\left(\bar{n}_{e}^{*}\right)-\mu\left(\underline{n}_{e}^{*}\right)\right]}
$$

Let's now focus on the marginal viewer. That is, the viewer who is indifferent between watching and not watching the media outlet's reports. Specifically, there will be two marginal viewers. One representing the most rightist citizen willing to watch news reports from a media outlet having an editor with idiosyncratic preferences $x_{e}$. The other one representing the most leftist citizen willing to watch such news reports. Hence, there will be a $\hat{x}_{e}=\hat{x}_{e}\left(x_{e}\right)$ and a $\tilde{x}_{e}=\tilde{x}_{e}\left(x_{e}\right)$ with $\hat{x}_{e}<\tilde{x}_{e}$ such that only citizens with $x_{i} \in\left[\hat{x}_{e}, \tilde{x}_{e}\right]$ will watch the news reports. ${ }^{26}$ In particular,

$$
\hat{x}_{e}=\frac{1}{2}-\delta\left(2 \mu\left(\bar{n}_{e}^{*}\right)-1\right)+\frac{C}{2 \operatorname{Pr}\left(n=\bar{n}_{e}^{*}\right)}
$$

\footnotetext{
${ }^{24}$ At the same time, from the citizens' perspective, it is equivalent whether the editor produces a coarse news report (e.g., endorsement) or she produces a news report showing all the signals (e.g., evidence) collected. Indeed, upon observing a coarse news report, citizens are able to infer which stopping threshold has been reached by the editor since they know the editor's idiosyncratic preferences. Moreover, this stopping threshold contains all the information needed by citizens to update their beliefs (i.e., the net difference of signals in favor of a candidate).

${ }^{25}$ These are simply the probabilities of hitting the two stopping thresholds in a stochastic process with two absorbing states (see Brocas and Carrillo, 2007). The online appendix provides a formal derivation of these probabilities.

${ }^{26}$ Notice that it could also be the case that $\hat{x}_{e}>\frac{1}{2}$ or $\tilde{x}_{e}<\frac{1}{2}$ but, clearly, not both.
} 
and

$$
\tilde{x}_{e}=\frac{1}{2}+\delta\left(1-2 \mu\left(\underline{n}_{e}^{*}\right)\right)-\frac{C}{2 \operatorname{Pr}\left(n=\underline{n}_{e}^{*}\right)}
$$

Furthermore, the following condition is assumed:

\section{Assumption}

$$
C<C^{\max }=\delta\left(\frac{1-\lambda^{\left.\bar{n}_{e}^{*}\right|_{x_{e}=\frac{1}{2}}}}{1+\lambda^{\left.\bar{n}_{e}^{*}\right|_{x_{e}=\frac{1}{2}}}}\right)
$$

where $\lambda=\frac{1-\theta}{\theta}$. It is easy to prove that when this assumption does not hold, there will never be any leftist or rightist citizen willing to watch any news report. The following lemma contains the main properties of the demand for news.

Lemma 1 Let $\left(\bar{n}_{e}^{*}, \underline{n}_{e}^{*}\right)$ be the optimal stopping rules of an editor with idiosyncratic preferences $x_{e}$. Then, $\left(\tilde{x}_{e}-\hat{x}_{e}\right)$ represents the demand for news of a media outlet with such an editor. This demand is decreasing in the opportunity cost of acquiring information (i.e., C). Moreover, this demand is increasing in:

1. The valence parameter (i.e., $\delta$ )

2. The threshold in the number of signals in favor of $R$ such that the editor stops acquiring information and endorses $R$ (i.e., $\bar{n}_{e}^{*}$ )

3. The threshold in the number of signals in favor of $L$ such that the editor stops acquiring information and endorses $L$ (i.e., $\left.\left|\underline{n}_{e}^{*}\right|\right)$.

At the same time, there is always an upper bound on the "extremism" of an editor above which the demand for news is strictly decreasing.

The above lemma summarizes the main features of the demand for news media by citizens. Hence, it represents the main building-block for all the results that will be obtained in the next section when discussing the optimal choice of editors by profit-maximizing media outlets within a given market structure (i.e., monopoly, duopoly or an arbitrary number of competing media outlets).

Obviously, a higher opportunity cost of watching news reports decreases the number of leftist and rightist citizens willing to watch such reports. Instead, the higher the valence component in the citizens utility function, the more leftist and rightist citizens will want to watch news. Hence, the more citizens care about knowing the state of the world, the more citizens will get informed. At the same time, all citizens care about receiving the most accurate information, i.e., the lower is $\underline{n}_{e}^{*}$ and the higher is $\bar{n}_{e}^{*}$, the more citizens will want to get informed. Indeed, all citizens who value information (i.e., the ones whose ex-post ranking of candidates is not always the same as their ex-ante one) would like to watch a media outlet having an editor who samples in both directions until infinity, since the more 
information she gets, the higher the citizens' expected utility. However, given the editor's cost of acquiring information and the opportunity cost that each citizen faces when accessing this information, when a citizen is choosing whether to watch a media outlet and/or choosing among alternative news media outlets, she takes into account how valuable - from her own perspective - the information collected by an editor are (in expectation). Overall, the model points out the presence of two rationales explaining why citizens find optimal to watch a media outlet whose editor has similar idiosyncratic preferences. For citizens with preferences $x_{i}<\left.\hat{x}_{e}\right|_{x_{e}=\frac{1}{2}}$ and $x_{i}>\left.\tilde{x}_{e}\right|_{x_{e}=\frac{1}{2}}$ only a media outlet with an editor with similar idiosyncratic preferences can be pivotal for their choice (i.e., they never find valuable the information coming from a moderate editor). Hence, either they will watch a media outlet with an editor with (sufficiently) similar preferences or they will not watch any media outlet at all.

On the other hand, citizens with preferences $x_{i} \in\left[\left.\hat{x}_{e}\right|_{x_{e}=\frac{1}{2}}, \frac{1}{2}\right) \cup\left(\frac{1}{2},\left.\tilde{x}_{e}\right|_{x_{e}=\frac{1}{2}}\right]$ find the information coming from a moderate editor valuable, but they may find the information coming from an editor with similar idiosyncratic preferences even more valuable. A citizen could make two types of errors. She may choose $L$ when $L$ is the low-quality candidate. Similarly, she may choose $R$ when $R$ is the low-quality candidate. A moderate citizen (i.e., $x_{i}=\frac{1}{2}$ ) cares about these two errors equally. Hence, she always prefers to watch a media outlet having a moderate editor since such an editor is the one whose news reports are, in expectation, most accurate (see Corollary 1). ${ }^{27}$ On the other hand, for example, a moderateleftist citizen cares more about not making the error of choosing $R$ when $s=l$. As shown by Corollary 1, a leftist editor has a lower probability of making such error but a higher probability of making a report in favor of $L$ when $s=r$ and a higher overall probability of making errors. Accordingly, a leftist citizen will then prefer a leftist editor to a moderate one. Therefore, rational citizens may prefer a media outlet with a like-minded editor simply because they derive a higher utility from the set of information acquired by such an editor with respect to the one acquired by a moderate editor. ${ }^{28}$

At the same time, as shown by Corollary 1, since the more ideological an editor is, the lower the expected accuracy of her news reports, there will always be an upper bound on the "extremism" of an editor above which the demand for news by rational citizens will be strictly decreasing.

Therefore, since $\tilde{x}_{e}$ is always increasing in $x_{e}$ for $x_{e} \leq 1 / 2$ and $\hat{x}_{e}$ is always increasing in $x_{e}$ for $x_{e} \geq 1 / 2$, this rational framework is able to explain the presence of preferences for like-minded sources of information. That is, the above lemma provides a rationale for the presence of a demand for news coming from ideological editors. In particular, while an economic rational for the demand for liked-minded news is already present in other models of

\footnotetext{
${ }^{27}$ Therefore, as a side result, the model also provides a rationale for why citizens with non-ideological preferences over candidates (i.e., moderate citizens) also prefer to watch news coming from a like-minded editor (i.e., a moderate editor).

${ }^{28}$ Durante and Knight (2012) analyze the demand for news in Italy. They show that, indeed, when the ideological position of a media outlet changes, viewers change their choice of news programs accordingly.
} 
demand-driven media bias (Mullainathan and Shleifer, 2005; Gentzkow and Shapiro, 2006; Annand et al., 2007; Chan and Suen, 2008), the mechanism underlying the above results is different from the existing literature. The results of Lemma 1 derive from the fact that, when choosing among different media outlets, rational citizens take into account that the news reports coming from media editors with different ideological preferences will be different simply because their optimal information acquisition strategies are different. At the same time, Lemma 1 also points out that rational citizens would never find optimal to demand news coming from editors having very extreme ideological preferences since the expected accuracy of such editors is very low. Hence, behavioral models (as the one of Mullainathan and Shleifer, 2005) remain probably better suited to explain the presence of a demand for news coming from extremist editors.

The following section analyzes the implications of such demand for news for the optimal choice of editors by profit maximizing media outlets.

\section{Optimal Choice of Editors by Media}

\subsection{Monopoly}

This section analyzes the implications of the citizen-editors model in a monopolistic market. The media outlet's owner wants to choose $x_{e}$ to maximize viewership. Choosing an editor from the population of citizens is analogous to choosing a "product" location on the $[0,1]$ line. Suppose the media outlet's owner chooses an editor with idiosyncratic preferences $x_{e}$. Then, the profit function is:

$$
\Pi\left(x_{e}, \hat{x}_{e}, \tilde{x}_{e}\right)=D\left(x_{e}, \hat{x}_{e}, \tilde{x}_{e}\right)=F\left(\tilde{x}_{e}\right)-F\left(\hat{x}_{e}\right)
$$

where $F\left(\tilde{x}_{e}\right)$ and $F\left(\hat{x}_{e}\right)$ are increasing functions of $x_{e}$. The following proposition characterizes under which conditions a profit-maximizing media outlet will choose a moderate editor and under which conditions it will choose an ideological one.

Proposition 2 Suppose there is just a monopolist profit-maximizing media outlet in the market for news. For any symmetric $f(x)$, then:

1. If the density of citizens' idiosyncratic preferences is increasing moving away from the "extremes" (i.e., $f(x)$ satisfies Condition A), then the media outlet will always choose a moderate editor (i.e., $x_{e}^{\text {mon }}=\frac{1}{2}$ ).

2. If the density of citizens' idiosyncratic preferences is increasing moving away from the median citizen (i.e., $f(x)$ satisfies Condition B), then the media outlet will always choose an ideological editor with preferences $x_{e}^{\text {mon }} \in\left[x_{e_{L}}^{\min }, \frac{1}{2}\right) \cup\left(\frac{1}{2}, x_{e_{R}}^{\max }\right]$ with $x_{e_{L}}^{\min }>0$ and $x_{e_{R}}^{\max }<1$. 
The above proposition shows that a monopolist media outlet will always choose a moderate editor when citizens are distributed uniformly or when the mass of moderate citizens is higher than the one of ideological ones (i.e., when Condition A applies). Instead, if the number of moderate citizens is lower than the one of ideological ones, the media outlet will prefer to choose an ideological editor (i.e., when Condition B applies). Indeed, in such a case the media outlet may increase its demand since many ideological citizens are willing to watch its news reports. At the same time, most moderate citizens will still want to acquire information from such a source rather than not acquiring any information at all.

Hence, when the media outlet is just maximizing profits, even though citizens do not derive any exogenous utility from biased information, the endogenous acquisition of costly information may induce a media outlet to choose an editor whose optimal information acquisition strategy is slanted in favor of the alternative ex-ante preferred by a subset of citizens (e.g., the rightists one).

However, even in this case the optimal editor will not be "too extremist". As shown by Corollary 1 , the more ideological an editor is, the lower the expected accuracy of her news reports. Hence, as suggested by Lemma 1, after some point choosing a more rightist (leftist) editor will decrease even the number of rightist (leftist) citizens willing to watch the media outlet, i.e., for $x_{e}>x_{e_{R}}^{\max }\left(x_{e}<x_{e_{L}}^{\min }\right)$.

\subsection{Duopoly}

Suppose now that $K=2$. That is, the market for news is composed of two profit maximizing media outlets. The following proposition summarizes the possible Nash equilibria that can arise in this case depending on the distribution of citizens' preferences. ${ }^{29}$

Proposition 3 Suppose there are two media outlets in the market for news. For any symmetric $f(x)$, then:

1. If the density of citizens' idiosyncratic preferences is increasing moving away from the "extremes" (i.e., $f(x)$ satisfies Condition A), then both media outlets choose moderate editors (i.e., $x_{e_{1}}^{\text {duop }}=x_{e_{2}}^{\text {duop }}=\frac{1}{2}$ ).

2. If the density of citizens' idiosyncratic preferences is increasing moving away from the median citizen (i.e., $f(x)$ satisfies Condition B), then $\exists C^{\text {Dev }}<C^{\max }$ such that:

(a) If $C>C^{\text {Dev }}$, then both media outlets choose moderate editors (i.e., $x_{e_{1}}^{\text {duop }}=x_{e_{2}}^{\text {duop }}=$ $\left.\frac{1}{2}\right)$

\footnotetext{
${ }^{29}$ Each citizen is implicitly assumed to watch at most one media outlet (which is, for example, the case when two television news programs broadcast at the same time or when there is an upper bound on the opportunity cost of watching news, e.g., time constraint). Nevertheless, as discussed in section A.3, this assumption is without loss of generality. If citizens were to acquire information from multiple sources, the incentives of media outlets to choose ideological editors would only be reinforced.
} 
(b) If $C<C^{D e v}$, then the two media outlets choose ideological editors having symmetric idiosyncratic preferences, i.e., $x_{e_{1}}^{\text {duop }}=1-x_{e_{2}}^{\text {duop }}$ where $x_{e_{1}}^{\text {duop }}, x_{e_{2}}^{\text {duop }} \in$ $\left[x_{e_{L}}^{\min }, \frac{1}{2}\right) \cup\left(\frac{1}{2}, x_{e_{R}}^{\max }\right]$ with $x_{e_{L}}^{\min }>0$ and $x_{e_{R}}^{\max }<1$. Moreover, the lower is $C$ the more ideological are the editors chosen by the media outlets.

When Condition A holds, despite the fact that by choosing, for example, a rightist editor a media outlet would increase the number of rightist citizens willing to watch its news (i.e., higher marginal rightist viewer), the net effect on the demand of choosing this editor rather than a moderate one would be always negative. Since choosing a less moderate editor also implies choosing an editor who will sample relatively less with respect to a more moderate one, the negative effect on moderate citizens' viewership would be higher than the positive effect on rightist citizens' viewership.

Moreover, even when Condition B holds, if the opportunity cost of acquiring information is high, the two media outlets will both choose moderate editors. This is the only case where a media outlet may not find it convenient to choose an ideological editor in a duopoly while it would in a monopoly. The reason behind this difference is that in the monopoly case choosing, for example, a rightist editor instead of a moderate one will decrease the demand for news by leftist citizens. However, moderate citizens will still be willing to watch such media outlet rather than not acquire any information at all. Instead, in the duopoly case, when the opportunity cost of acquiring information is high, by choosing a rightist editor, a media outlet may face a reduction in the demand for its news by moderate citizens larger than the increase in the demand by rightist citizens.

On the other hand, when the opportunity cost is low, the demand for news by extremist citizens will be high enough to induce media outlets to choose ideological editors. Thus, the two media outlets will end up choosing symmetric types of ideological editors. That is, while in the monopolistic case there was only a rightist (or leftist) editor, in presence of two media outlets there will be also a leftist (or rightist) editor. Moreover, the lower is the opportunity cost, the higher will be the difference between the idiosyncratic preferences of the editors chosen by the two media outlets. Finally, given the results of Lemma 1, even in this case optimal editors could never be "too extremist".

\subsection{Multiple Media Outlets}

This section analyzes the case where there are multiple media outlets in the market for news, i.e., $K>2$. The above analysis has shown that when moderate citizens are uniformly distributed in the policy space, or when the mass of moderate citizens is higher than the one of ideological citizens, media outlets will choose moderate editors both in a monopoly and in a duopoly. The following proposition shows that when there are multiple media outlets in the market for news, this is not always the case. Specifically, when $x_{i} \sim U[0,1]$, as the number of media outlets present in the market increases, the equilibrium where every media 
outlet chooses a moderate editor is not sustainable anymore. Indeed, any media outlet would have an incentive to differentiate its "news product" by choosing an ideological editor.

Proposition 4 Suppose that citizen's idiosyncratic preferences are distributed uniformly in $[0,1]$. Then, it exists a threshold in the number of media outlets present in the market for news $K^{*} \in(2, \infty)$ such that for $K>K^{*}$ the set $\left\{x_{e_{j}}=\frac{1}{2}, \forall j=1, \ldots, K\right\}$ is not an equilibrium. In such case, it still exists a symmetric mixed-strategy Nash equilibrium. Moreover, $K^{*}$ is increasing in $C$.

The above proposition shows that when the market for "moderate news" gets crowded, media outlets will prefer to choose a different location for their news product. That is, the higher the degree of competition in the market for news, the more likely it is that media outlets will choose ideological editors. This result is typical in the literature on demanddriven media bias (e.g., Mullainathan and Shleifer, 2005; Annand et al., 2007; Chan and Suen, 2008). It arises from the incentives of profit-maximizing media outlets to differentiate their news products.

This theoretical mechanism is consistent with the emerging empirical evidence comparing the degree of ideological polarization of news sources in the online market for news with respect to the offline media market, e.g., online newspapers and blogs with respect to traditional newspapers and TV. Indeed, the higher number of competing media outlets present in the online market for news seems to be associated with a higher degree of ideological polarization with respect to the offline market for news (Sunstein, 2007; Gentzkow and Shapiro, 2011). At the same time, the theoretical insights of Proposition 4 are also in line with the empirical analysis by Gentzkow et al. (2012) on the US press industry in the early 20th century, showing that economic competition enhances ideological diversity.

Moreover, since the higher the opportunity cost of acquiring information, the less extremists citizens will find it optimal to acquire information, as such cost increases the likelihood of media outlets choosing ideological editors decreases. ${ }^{30}$ That is, it is possible to reinterpret the above proposition with respect to $C$. For a given $K>2$, there will exist a $C^{*}(K)$ such that for $C>C^{*}(K)$, all media outlets will choose a moderate editor from the population of citizens. Instead, for $C<C^{*}(K)$, media outlets will choose ideological editors. This result, along with the ones of Propositions 2 and 3, suggests that more moderate editors should be expected to prevail in a news market where the opportunity cost is high. A clear application of this result is represented by the differences between the broadcast media sector with respect to the press. The opportunity cost of watching a report from a broadcast media is arguably lower than the one of reading a newspaper. The analysis thus suggests that, all other things equal, more moderate editors should be present in the press sector than in the broadcast media sector. At the same time, there should be more extremist citizens watching

\footnotetext{
${ }^{30}$ Indeed $\lim _{C \rightarrow C^{\max }} K^{*} \rightarrow \infty$.
} 
broadcast media and a higher overall demand for broadcast media with respect to the one faced by the press.

\section{Discussion: Information and Welfare}

\subsection{Private Value of Information and Utility}

As usual in the literature on the demand for news (e.g., Strömberg, 2004b; Mullainathan and Shleifer, 2005; Gentzkow and Shapiro, 2006; Chan and Suen, 2008; Anderson and McLaren, 2012), I have assumed that citizens receive utility from choosing a given candidate/alternative per se. ${ }^{31}$ Since news has a public-good nature and the probability of being pivotal is close to zero, the expected benefit of acquiring information is likely to be negligible. That is, acquiring information is a typical free-riding problem. Hence, in my model, as in the rest of this literature, it is necessary to explain why citizens bother spending the opportunity cost of watching TV news or reading newspapers.

A straightforward rationale for the demand for news is the one proposed by Strömberg (2004b) and Anderson and McLaren (2012). That is, citizens may be using news reports to decide on a private action whose value depends on the public policy implemented (or candidate elected). For example, the news could cover the quality and virtues of the public school system and the private decision is the choice between enrolling in a public or in a private school. That is, the willingness to acquire information on the state of the world "to make a better private decision generates a market demand for news, and this then through the voting system affects the direction of the public decision." (Anderson and McLaren 2012, page 834$).{ }^{32}$

\subsection{Citizens' welfare}

Given that voting is costless and taking into account the rationale for information acquisition explained in the previous section, every citizen would turnout and choose the candidate which yields her the higher expected utility (given her posterior beliefs). Therefore, it is possible to compare citizens' welfare in an equilibrium where only moderate editors are chosen by media outlets (e.g., a duopoly where $f(x)$ satisfies Condition B and $C>C^{D e v}$ ) with one where media outlets choose ideological editors (e.g., a a duopoly where $f(x)$ satisfies Condition B and $C<C^{D e v}$ ). Specifically, in order to assess citizen's welfare, it is necessary to analyze how the expected utility of different segment of citizens may vary in presence of

\footnotetext{
${ }^{31}$ Similarly, the model shares with this literature the implicit assumption that a citizen must watch the news report in order to learn its information content.

${ }^{32}$ Alternatively, the citizens' decision to become informed may be seen as deriving from an intrinsic psychological benefit from choosing the high-quality candidate as in Chan and Suen (2008). Larcinese (2009) presents a decision-theoretical model of voters' demand for political information. See also Piolatto and Schuett (2011) for a model of the demand for news by ethical voters.
} 
moderate or ideological editors. It is straightforward to see that a subset of citizens may be better off in an equilibrium where media outlets choose ideological editors with respect to one where only moderate editors are present. Indeed, as shown in Lemma 1 and discussed in Section 4, the presence of ideological editors allows a fraction of the population (i.e., leftist and rightist citizens) to find a valuable source of information and another one (i.e., moderateleftist and moderate-rightist) to choose a source of information yielding a higher expected utility. At the same time, as shown by Corollary 1, the accuracy of an ideological editor's news report is lower than the one of a moderate editor. Moderate citizens will then be worse off in such a equilibrium where media outlets choose ideological editors since the news reports coming from these type of editors involve a higher expected probability of endorsing the lowvalence candidate. Considering an utilitarian citizens' welfare function, the overall effect of having an equilibrium with ideological editors will then crucially depend on the shape of the distribution of citizens' ideological preferences. In particular, as shown by Proposition 3 , in a duopoly where $f(x)$ satisfies Condition B media outlets choose (symmetric) ideological editors when $C<C^{D e v}$ while they both choose moderate editors when $C \geq C^{\text {Dev }}$. Hence, in presence of a density function $f(x)$ which satisfies Condition $\mathrm{B}$, the overall welfare will be higher in a duopoly where $C<C^{D e v}$ with respect to a situation where $C \geq C^{D e v}$ since $f(x)$ is such that most citizens have ideological preferences close to either the upper or lower bound of the distribution.

\section{Conclusions}

The paper has analyzed a market for news in which profit maximizing media outlets choose their editors from the population of citizens. The results identify a novel mechanism of media bias: the bias in a media outlet's news reports is the result of the slanted optimal information acquisition strategy of its editor. Accordingly, the analysis has shown that the editors' endogenous information acquisition results in rational citizens finding it optimal to choose a like-minded source of information (i.e., watch a media outlet having an editor with similar idiosyncratic preferences). Indeed, citizens may obtain a higher expected utility from the set of information acquired by a like-minded editor with respect to the one acquired by a moderate editor. Hence, even though citizens do not derive any exogenous utility from biased information, they all share the same prior beliefs and media outlets are just maximizing profits, the endogenous acquisition of costly information may induce a media outlet to choose an editor whose optimal information acquisition strategy is "slanted" in favor of the alternative ex-ante preferred by a subset of citizens. Therefore, my model provides a novel rationale for the presence of slant in the market for news purely based on the citizens' demand for the most valuable source of information. At the same time, the results also show that there is always an upper bound on the possible "extremism" of an editor above which the demand for news by rational citizens is strictly decreasing. 
Similarly to other models of demand-driven media bias (e.g., Mullainathan and Shleifer, 2005; Annand et al., 2007; Chan and Suen, 2008), the results show that the higher the degree of competition in the market for news, the more likely that media outlets will choose ideological editors. That is, when the market for news gets crowded, a media outlet may prefer to differentiate its news product by choosing a different location in the policy space (i.e., choose an editor with different idiosyncratic characteristics), rather than sharing the demand for news of moderate citizens with the other media outlets. This theoretical mechanism is consistent with the higher degree of ideological polarization of news media observed in the online market for news with respect to the offline market for news (Sunstein, 2007; Gentzkow and Shapiro, 2011) and with historical evidence on the US newspapers industry (Gentzkow et al., 2012)

At the same time, the model is also particularly fit to capture the behavior of the socalled citizen-journalists. As pointed out by Battaglini (2013), with the emergence of new online media platforms (e.g., blogs) citizens are taking an active role in the production of news. Hence, this theoretical mechanism sheds light on how citizens acquire informationaccording to their ideological preferences - when acting as journalists in online news media platforms. Moreover, if the valence component of citizen-journalists were to be lower with respect to the one of citizen-editors employed by traditional news media (e.g., due to different reputation incentives), the results would suggest that the expected accuracy of news reports by traditional news media are likely to be higher than the one of new news media. This is an issue left for future research.

More generally, this paper has focused only on the demand for slanted news. In order to carefully assess the effects of competition on citizens' welfare, policy regulators should take into account the possible presence of both demand-driven and supply-driven sources of media bias in the market for news. 


\section{References}

[1] Anand, B., Di Tella, R., and Galetovic, A. 2007. "Information of Opinion: Media Bias as Product Differentiation." Journal of Economics \& Management Strategy, 16(3): 635-682.

[2] Alterman, E. 2003. What liberal media? The truth about bias and the news. New York: Basic Books.

[3] Anderson, S., P., and McLaren, J. 2012. "Media Mergers and Media Bias with Rational Consumers." Journal of the European Economic Association, 10(4): 831-859.

[4] Aragones E., and Palfrey T., R. 2002. "Mixed Equilibrium in a Downsian Model with a Favored Candidate." Journal of Economic Theory, 103: 131-161.

[5] Bagdikian, B. H. 2004. The new media monopoly. Boston: Beacon Press.

[6] Baron, D., P. 2006. "Persistent Media Bias." Journal of Public Economics, 90(1): 1-36.

[7] Battaglini, M. 2013. "Comments on Prat and Strömberg, and Robinson and Torvik." In Advances in Economics and Econometrics: Theory and Applications, Tenth World Congress, forthcoming.

[8] Besley, T., and Coate, S. 1997. "An Economic Model of Representative Democracy." The Quarterly Journal of Economics, 112(1): 85-114.

[9] Besley T., and Prat A. 2006. "Handcuffs for the grabbing hand? Media capture and government accountability." American Economic Review, 96(3): 720-736.

[10] Brocas, I. and Carrillo, J., D. 2007. "Influence through Ignorance." RAND Journal of Economics, 38(4): 931-947.

[11] Brocas, I. and Carrillo, J., D. 2009. "Information acquisition and choice under uncertainty." Journal of Economics and Management Strategy, 18(2): 423-455.

[12] Brocas, I., Carrillo, J., D. and Palfrey, T., R. 2011. "Information Gatekeepers: Theory and Experimental Evidence." Economic Theory, forthcoming.

[13] Calvert, R. L. 1985. "The Value of Information: A Rational Choice Model of Political Advice." Journal of Politics, 47: 530-55.

[14] Chan, J., and Suen, W. 2008. "A Spatial Theory of News Consumption and Electoral Competition." The Review of Economic Studies, 75(3): 699-728.

[15] Chiang, C., F., and Knight, B., G. 2011. "Media Bias and Influence: Evidence from Newspaper Endorsements." The Review of Economic Studies, 78(3): 795-820.

[16] Cukierman, A., and Tommasi, M. 1998. "When Does it Take a Nixon to Go to China?" American Economic Review, 88(1): 180-197.

[17] Dasgupta, P. and Maskin, E. 1986. "The Existence of Equilibrium in Discontinuous Economic Games II: Applications." The Review of Economic Studies, 53(1): 27-41.

[18] Davies, N. 2008. Flat Earth News. London: Vintage.

[19] DellaVigna, S. and Gentzkow, G. 2010. "Persuasion: Empirical Evidence." Annual Review of Economics, 2: 643-69.

[20] DellaVigna, S. and Kaplan, E. 2007. "The Fox News Effect: Media Bias and Voting." The Quarterly Journal of Economics, 122(3): 1187-1234. 
[21] Drago, F., Nannicini, T., and Sobbrio, F. 2013. "Meet the Press: How Voters and Politicians Respond to Newspaper Entry and Exit." IZA Discussion Paper 7169.

[22] Durante R. and Knight B. 2012. "Partisan Control, Media Bias, and Viewer Responses: Evidence from Berlusconi's Italy", Journal of the European Economic Association, 10(3): 451-481.

[23] Ellman, M. and Germano, F. 2009. "What do the Papers Sell? A Model of Advertising and Media Bias." Economic Journal, 119: 680-704.

[24] Eisensee, T., and Strömberg, D. 2007. "News Floods, News Droughts, and U.S. Disaster Relief." Quarterly Journal of Economics, 122(2).

[25] Enikolopov, R., Petrova, M., and Zhuravskaya, E. 2011. "Media and Political Persuasion: Evidence from Russia." American Economic Review, 101(7): 3253-85.

[26] Gentzkow, M. 2006. "Television and voter turnout." Quarterly Journal of Economics 121(3), 931-972.

[27] Gentzkow M., and Shapiro J. 2006. "Media Bias and Reputation." Journal of Political Economy, 114(2): 280-316.

[28] Gentzkow M., and Shapiro J. 2010. "What Drives Media Slant? Evidence from U.S. Daily Newspapers.", Econometrica, 78(1): 35-71.

[29] Gentzkow M., and Shapiro J. 2011. "Ideological Segregation Online and Offline." The Quarterly Journal of Economics, 126 (4): 1799-1839.

[30] Gentzkow M., Shapiro J. and Sinkinson, M. 2011. "The Effect of Newspaper Entry and Exit on Electoral Politics." American Economic Review, 101(7): 2980-3018.

[31] Gentzkow M., Shapiro J. and Sinkinson, M. 2012. "Competition and Ideological Diversity: Historical Evidence from US Newspapers." NBER Working Paper 18234.

[32] Gerber, A., Karlan, D., S. and Bergan, D. 2009. "Does the Media Matter? A Field Experiment Measuring the Effect of Newspapers on Voting Behavior and Political Opinions." American Economic Journal: Applied Economics, 1(2): 35-52.

[33] Goldberg, B. 2002. Bias: A CBS insider exposes how the media distort the news. Washington, DC: Regency Publishing, Inc.

[34] Groseclose, T. and Milyo, J. 2005. "A Measure of Media Bias." The Quarterly Journal of Economics, 120(4): 1191-1237.

[35] Ho, D. E., and Quinn, K. M. 2008. "Measuring Explicit Political Positions of Media." Quarterly Journal of Political Science, 3: 353-377.

[36] Kamenica, E., and Gentzkow, M. 2011. "Bayesian Persuasion." American Economic Review, 101: $2590-2615$.

[37] Larcinese, V. 2009. "Information Acquisition, Ideology and Turnout: Theory and Evidence from England." Journal of Theoretical Politics, 21(2): 237-276.

[38] Larcinese, V., Puglisi, R., and Snyder, Jr., J. M. 2011. "Partisan bias in economic news: Evidence on the agenda-setting behavior of U.S. newspapers." Journal of Public Economics, 95(9-10): 1178-1189.

[39] Li, H., and Suen, W. 2004. "Delegating Decisions to Experts." Journal of Political Economy, 112(1): 311-335. 
[40] Mullainathan S., and Shleifer A. 2005. "The Market for News." American Economic Review, 95(4): 1031-1053.

[41] Oberholzer-Gee, F. and Waldfogel, J. 2009. "Media markets and localism: Does local news en Español boost Hispanic voter turnout?" American Economic Review, 99(5): 2120-28.

[42] Osborne M., J., and Slivinsky A. 1996. "A Model of Political Competition with CitizenCandidates." The Quarterly Journal of Economics, 111(1): 65-96.

[43] Patterson, T., E., and Wolfgang, D. 1996. "News Decisions: Journalists as Partisan Actors." Political Communication, 13: 455-468.

[44] Pew Research Center. 2012. "Further Decline in Credibility Ratings for Most News Organizations". Pew Research Center for the People and the Press. August 16, 2012.

[45] Piolatto, A., and Schuett, F. 2011. "Ethical voters and the demand for political news." Working paper, Barcelona Institute of Economics (IEB) and Tilburg University.

[46] Prat, A., and Strömberg, D. 2013. "The Political Economy of Mass Media." In Advances in Economics and Econometrics: Theory and Applications, Tenth World Congress, forthcoming.

[47] Puglisi, R. and Snyder, Jr., J. M. 2011. "The Balanced U.S. Press." NBER Working Papers 17263, National Bureau of Economic Research.

[48] Shiryaev, A., N. 2007. Optimal Stopping Rules, Springer, Berlin.

[49] Snyder, J., and Strömberg, D. 2010. "Press Coverage and Political Accountability." Journal of Political Economy, 118 (2).

[50] Strömberg, D. 2004a. "Radio's Impact on Public Spending." Quarterly Journal of Economics, 119(1): $189-221$.

[51] Strömberg, D. 2004b. "Mass Media Competition, Political Competition, and Public Policy." The Review of Economic Studies, 71(1): 265-284.

[52] Sunstein, C., R. 2007. Republic.com 2.0. Princeton (NJ): Princeton University Press. 


\section{Appendix A}

\section{A.1 Media owners and citizen-editors}

Since the main focus of the paper is on the demand for slanted news, the model provides a stylized representation of media outlets' profits. Considering a more general compensation mechanism for the editor would affect both the revenues and the costs of a media outlet. Once on the job, editors (and journalists) are the ones who will spend time and exert effort to collect evidence on any given issue. That is, media outlets/media owners do not directly bear this day to day cost of information acquisition. Nevertheless, in order to increase its profits, a media outlet may try to induce its editor to change her optimal information acquisition strategy by designing an incentive mechanism. As shown by Lemma 1, ideally all citizens would like to watch a media outlet whose editor keeps acquiring information until she learns the true state of the world (i.e., $\left.\underline{n}_{e}^{*}=-\infty, \bar{n}_{e}^{*}=\infty\right)$. However, it is not feasible for the media outlet to induce the editor to adopt such a sampling strategy. This is true for two simple reasons: $i$ ) information acquisition is costly for the editor and hence it is also costly for the media outlet to compensate the editor for acquiring extra pieces of information; $i i)$ the media outlet cannot monitor the information gathered by the editor (i.e., the media outlet cannot observe the draws sampled by the editor). Nevertheless, a media outlet may induce an editor to choose stopping rules which are higher (in absolute value) with respect to the ones she would choose in the absence of any incentive mechanism. In this perspective, a simple incentive mechanism that the media outlet could implement is to offer to the editor a share $\alpha$ of the media outlet's profits. This would induce the editor to choose higher (in absolute value) stopping rules. Indeed, in the absence of perfect monitoring, an incentive scheme rewarding the editor for each extra piece of evidence collected would produce the same results of a decrease in the marginal cost of sampling $c$ (i.e., any signal acquired is more valuable or, equivalently, less costly). That is, as shown by Proposition 1, a lower $c$ induces an editor to acquire more information. ${ }^{33}$ Similarly, the media outlet (or, more generally, the market for news) may provide an editor with a "reputation premium" when her news reports turn out to be accurate (i.e., when endorsing the high-valence candidate). That is, the editor may receive an extra positive payoff when her choice over candidates match the true state of the world. It is immediate to see how such an incentive mechanism is equivalent to increasing the value of the valence parameter $\delta$ in the editor's utility function. Hence, as shown by Proposition 1, the presence of a "reputation premium" would induce editors to acquire more information before producing a news report.

Therefore, incentive mechanisms aimed at decreasing the (net) marginal cost of sampling or at increasing the editor's valence parameter would, indeed, increase the informativeness of the editor's news reports. Nevertheless, such incentive mechanisms would not change the main results of the model since the stopping rules of ideological editors would still be asymmetric. Indeed, as shown by Proposition 1, the presence of a private value component in the editor's utility function always results in an ideological editor adopting a slanted information acquisition strategy. ${ }^{34}$

Moreover, it would be extremely costly for a media outlet to induce a moderate editor to gather an amount of information such that even extremists citizens would consider this media outlet a valuable source of information. ${ }^{35}$ In addition, as discussed in section 4 , while all citizens with preferences $\left.\hat{x}_{e}\right|_{x_{e}=\frac{1}{2}}<x_{i}<\left.\tilde{x}_{e}\right|_{x_{e}=\frac{1}{2}}$ find the information coming from a moderate editor valuable,

\footnotetext{
${ }^{33}$ Notice that a media outlet may also decrease $c$ by giving the editor more resources to produce the news reports (e.g., more correspondents, better technology, more resources to investigate an issue, etc.).

${ }^{34}$ Moreover, the cost of acquiring information by editors may be also reinterpreted as a discount factor (see Brocas and Carrillo 2009). In such case, each editor has to decide when to stop gathering information. Hence, by inducing an editor to sample more, a media outlet would also delay the release of the news report which may have a negative effect on the demand for it and, hence, on the profits.

${ }^{35}$ Indeed, $\hat{x}_{e} \rightarrow 0$ and $\tilde{x}_{e} \rightarrow 1$ if and only if $\underline{n}_{e}^{*} \rightarrow-\infty, \bar{n}_{e}^{*} \rightarrow \infty, \delta \rightarrow 1 / 2$ and $C \rightarrow 0$.
} 
some of them would find the information coming from an editor with similar idiosyncratic preferences even more valuable. Hence, there will always be a demand for "slanted" news by ideological citizens that a media outlet may capture by simply hiring an ideological editor. ${ }^{36}$

\section{A.2 Editor's Influence on Citizens}

In the model the utility of the editor depends on her own choice. Nevertheless, even if the editor's utility were to depend on the citizens' choice, the information acquisition strategy of the editor would not change. Indeed, the only credible strategy by an editor with idiosyncratic preferences $x_{e}$ is to report $\underline{n}_{e}^{*}$ upon reaching $\underline{n}_{e}^{*}$ and to report $\bar{n}_{e}^{*}$ upon reaching $\bar{n}_{e}^{*}$. A parallel to the politicaleconomy literature on citizens-candidates (Osborne and Slivinsky, 1996; Besley and Coate, 1997), might be particularly useful to exemplify the rationale behind this mechanism. In the citizencandidate framework, candidates could in principle gain votes by proposing a platform that is more appealing to a larger number of voters with respect to her own preferred policy. However, this is not a feasible strategy for a candidate simply because voters know that the citizen-candidate could only credible commit to her preferred policy since, once in office, this is the policy that she will pick. Similarly, a citizen-editor is only able to commit to her own stopping thresholds since these are the only ones that could be credible from the viewers' point of view. That is, the stopping thresholds represent the evidence that an editor requires to be "convinced" about a given candidate. Any additional evidence that the editor might claim to have found in favor of her "endorsed" candidates will simply not be credible. Indeed, since citizens know the idiosyncratic preferences of the editor, even if she were to try to influence citizens' choice by over-reporting the number of signals in favor of a given candidate, citizens would still be able to perfectly discount her "bias" and infer the actual stopping threshold (i.e., any $n>\bar{n}_{e}^{*}$ would be interpreted as $\bar{n}_{e}^{*}$ and any $n<\underline{n}_{e}^{*}$ as $\underline{n}_{e}^{*}$ ). Suppose for example that a leftist editor would announce that she would choose an optimal information acquisition rule (stopping threshold) which is "tougher" on the leftist candidate. That is, she announces that she would require more signals in favor of $L$ in order to endorse such candidate. This potentially may lead more individuals to be willing to read her news reports since this would be more informative. However, such announcement would simply not be credible. Indeed, readers cannot monitor the information acquired by editors/journalists. Since the endorsement/report is based on the difference between the number of signals in favor of $L$ with respect to the ones in favor of $R$ (i.e., net evidence), readers know that the editor may simply manipulate the reported number of signals in favor of one candidate or the other. Suppose, for example, that the stopping rule of a leftist editor is such that she would endorse candidate $L$ after having found $x$ more signals in favor of $L$ and, instead, she announces that she will endorse $L$ only after having found $y$ more signals in favor of $L$ (with $y>x$ ). Then, as in any model of supply-driven media bias, such an editor may simply selectively omit a subset of the available information once she has reached one of her optimal stopping thresholds. That is, once she arrives to the " $x$ more in favor of $L$ " threshold she may easily show to readers " $y$ more in favor of $L$ " by simply hiding $(y-x)$ signals in favor of $R$. Therefore, readers would not find credible such announcement by a media editor. Consequently, the media editor is only able to credibly commit to her own optimal information acquisition strategy.

Notice that the model could indeed be seen as a special case of a commitment-free mechanism of Bayesian persuasion, as defined by Kamenica and Gentzkow (2011), where the Sender (the editor) can influence the choice of a rational Bayesian Receiver (the citizens) by influencing her beliefs. Specifically, in my setting the fact that the Sender's preferences depend on the state of the

\footnotetext{
${ }^{36}$ Moreover, it would be cheaper for a media outlet to capture such demand for "slanted" news of nonmoderate citizens by hiring an editor with similar idiosyncratic preferences, rather than hiring a moderate one and provide her with incentives to acquire a large amount of information in both directions.
} 
world and acquiring signals is costly, mitigates the incentive compatibility constraints. That is, there is an endogenous commitment mechanism arising from the editor's idiosyncratic preferences and the cost of drawing a signal. The Receiver knows that the only credible signal realization is the one implicitly defined by the two stopping thresholds of the Sender (i.e., the editor can only credibly commit to such signal acquisition strategy). Any other mechanism would, simply, not be credible. The stopping thresholds represent the net difference in the number of signals in favor of one candidate. Hence, once the editor has reached one of the two thresholds, she has always an incentive to hide signals against the endorsed candidate. Hence, since there is an alignment of preferences between the Sender and the Receiver (i.e., all citizens willing to acquire information from a given editor will have the same ex-post ranking of preferences as the one of the editor), the Sender will truthfully reveal the signal realization.

Clearly, in the presence of uncertainty on the editor's idiosyncratic preferences there would also be uncertainty on the editor's optimal stopping thresholds. That is, if citizens only knew that $x_{e} \sim g(x)$ with $\operatorname{supp}(x)=\left[x_{e}^{A}, x_{e}^{B}\right]$ and $x_{e}^{A}<x_{e}^{B}$, then they would also know that $\underline{n}_{e}^{*} \sim g\left(\underline{n}_{e}^{*}\left(x_{e}\right)\right)$ with $\operatorname{supp}\left[g\left(\underline{n}_{e}^{*}\left(x_{e}\right)\right)\right]=\left[\underline{n}_{e}^{B}, \underline{n}_{e}^{A}\right]$ where $\underline{n}_{e}^{B}=\underline{n}_{e}^{*}\left(x_{e}^{B}\right)<\underline{n}_{e}^{A}=\underline{n}_{e}^{*}\left(x_{e}^{A}\right)$, since there is a one-toone mapping between preferences and optimal stopping thresholds. Similarly, $\bar{n}_{e}^{*}\left(x_{e}\right) \sim g\left(\bar{n}_{e}^{*}\left(x_{e}\right)\right)$ with $\operatorname{supp}\left[g\left(\bar{n}_{e}^{*}\left(x_{e}\right)\right)\right]=\left[\bar{n}_{e}^{B}, \bar{n}_{e}^{A}\right]$ where $\bar{n}_{e}^{B}=\bar{n}_{e}^{*}\left(x_{e}^{B}\right)<\bar{n}_{e}^{A}=\bar{n}_{e}^{*}\left(x_{e}^{A}\right)$. In presence of such additional source of uncertainty, the editor will have an incentive to over-report signals in favor of the preferred candidate once she has reached one of the two stopping thresholds. That is, such uncertainty would introduce in the model a "supply-driven" bias in news reports since the editor would have an incentive to bias its news reports by selectively omitting a subset of her information. Nevertheless, if the editor had to report $\bar{n}_{e}^{A}$, citizens' posterior beliefs would be $\mu\left(\bar{n}_{e}^{A}\right)=\mu\left(E\left(\bar{n}_{e}^{*}\left(x_{e}\right) \mid \bar{n}_{e}^{A}\right)\right) .{ }^{37}$ That is, citizens will still be able to infer the interval in which the optimal editor's stopping threshold lies and discount their posterior beliefs accordingly. Hence, the main mechanism and intuition of the model would not change. Obviously, the more ideologically distant from the endorsed candidate the editor is believed to be, the more influential her reports will be. In other words, the editor's endorsement will be stronger: $i$ ) the more moderate the editor is believed to be, upon endorsing the ideologically closer candidate; $i i)$ the less moderate the editor is believed to be, upon endorsing the ideologically least preferred candidate. Hence, in most of the cases (i.e., when endorsing the ideologically closer candidate), an editor would like to be believed to be as "unbiased" (i.e., moderate) as possible. Indeed, consistent with the theoretical predictions of the model, the empirical analysis of Chiang and Knight (2011) shows that the degree of influence of a newspaper on voters depends on the "credibility" of the endorsement.

\section{A.3 Multiple Sources of Information}

Throughout the analysis, it was assumed that citizens watch at most one media outlet. Nevertheless, while such assumption greatly simplifies the analysis, the intuition and the main results of the model do not rely on it. Indeed, if citizens were to acquire information from multiple sources, the incentives of media outlets to choose ideological editors would only be reinforced. For any citizen, watching two media outlets with a moderate editor has the same value of watching only one. Specifically, after having observed the news report of a moderate editor, watching an additional media outlet with another moderate editor would either not change the citizen's ranking of preferences, or it would lead citizen's posterior beliefs to be equal to the prior (i.e., the two reports would just "cancel" each other). Hence, if citizens could access multiple sources of information, the incentives of media outlets to differentiate their products by hiring ideological editors would, indeed, be higher.

\footnotetext{
${ }^{37}$ Similarly, upon reporting $\bar{n}_{e}^{B}$, citizens' posterior beliefs would be $\mu\left(\bar{n}_{e}^{B}\right)=\mu\left(E\left(\bar{n}_{e}^{*}\left(x_{e}\right) \mid \bar{n}_{e}^{B}\right)\right)$.
} 


\section{Appendix B}

The following graph illustrates the relationship between the perceived quality and fairness of US news organizations from 1985 to 2011 (Pew Research Center, 2012). In particular, the perceived quality of news organizations, in a given year, is measured in terms of the percentage of individuals who think that news organizations get their facts straight. The perceived fairness of news organizations is measured as the percentage of individuals who think that news media deal fairly with all sides (on political and social issues).

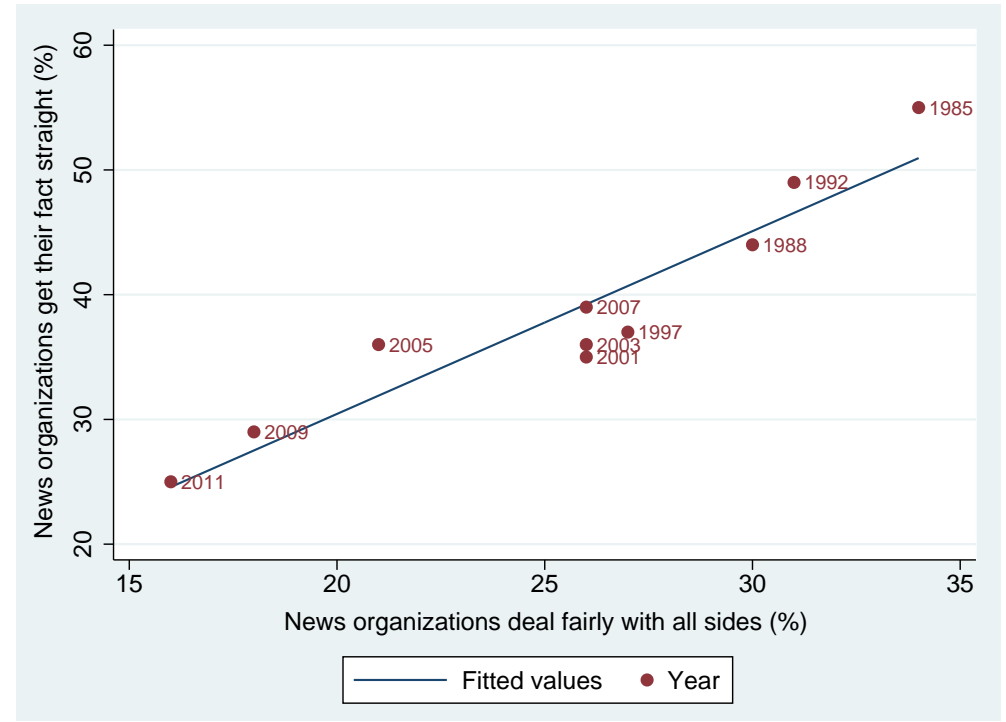

Fig. B1. Perceived quality and fairness of US news organizations.

(Source: Pew Research Center, 2012)

The following graph illustrates the evolution of perceived quality and fairness of US news organizations over time.

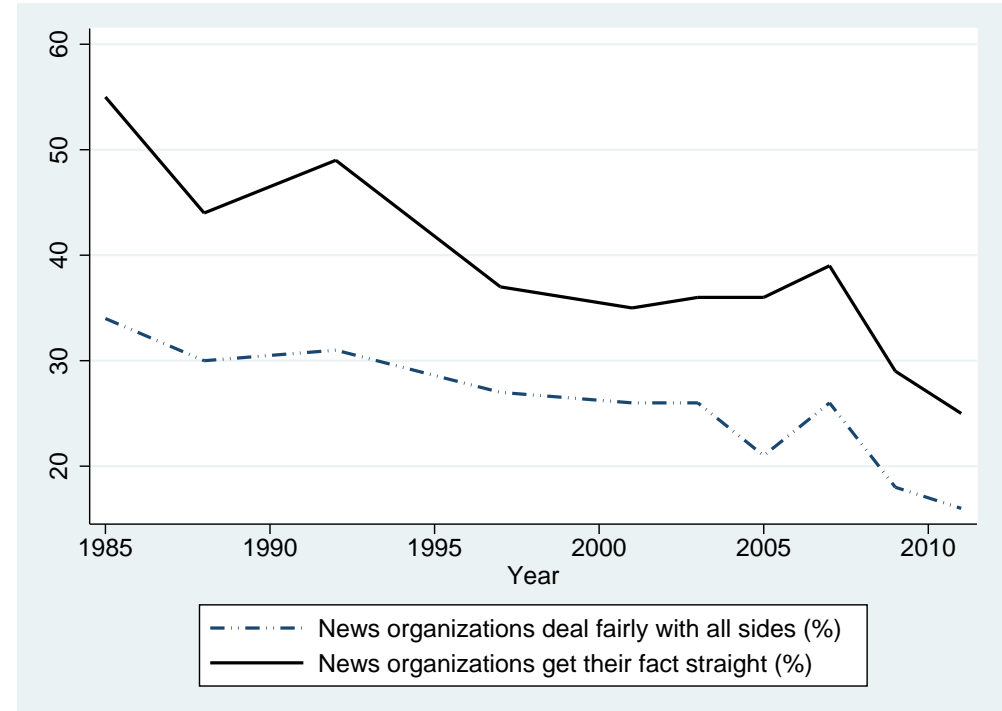

Fig. B2. Evolution of perceived quality and fairness of news organization in the US

(Source: Pew Research Center, 2012)

The above graphs present evidence consistent with the theoretical prediction of Corollary 1. That is, moderate media editors (i.e., the ones having a "fair and balanced" information acquisition strategy) are the ones whose news reports are the most accurate (in expectation). 


\section{Appendix C}

\section{Proof of Proposition 1}

The problem involves analyzing a stochastic process with two absorbing states. Specifically, the equations characterizing these two absorbing states (i.e., $\underline{n}_{e}^{*}$ and $\bar{n}_{e}^{*}$ ) must be determined. After $m$ draws, given that a current difference in signals in favor of $r$ equal to $n$, the value function of editor $e$ is given by (9). This is a standard problem of sequential testing of two simple hypotheses (see Chapter 4 in Shiryaev, 2007). Hence, it can be proven that $\bar{n}_{e}^{*}$ and $\underline{n}_{e}^{*}$ are defined implicitly by the following two first order conditions: ${ }^{38}$

$$
\begin{gathered}
\left.\frac{\partial V_{e}}{\partial \bar{n}_{e}^{*}}\right|_{\bar{n}_{e}^{*}}=\frac{(\ln \lambda) \lambda^{\bar{n}_{e}^{*}}}{\lambda \underline{n}_{e}^{*}-\lambda^{\bar{n}_{e}^{*}}}\left[(2 x-1)\left(\lambda^{\underline{n}_{e}^{*}}+1\right)-\left(\lambda^{\underline{n}_{e}^{*}}-1\right)\left(2 \delta-H\left(\bar{n}_{e}^{*}-\underline{n}_{e}^{*}\right)\right)\right]-H\left(1-\lambda^{\bar{n}_{e}^{*}}\right)=0 \\
\left.\frac{\partial V_{e}}{\partial \underline{n}_{e}^{*}}\right|_{n=\underline{n}_{e}^{*}}=\frac{(\ln \lambda) \lambda^{\underline{n}_{e}^{*}}}{\lambda^{\underline{n}_{e}^{*}}-\lambda^{\bar{n}_{e}^{*}}}\left[(2 x-1)\left(\lambda^{\bar{n}_{e}^{*}}+1\right)+\left(1-\lambda^{\bar{n}_{e}^{*}}\right)\left(2 \delta-H\left(\bar{n}_{e}^{*}-\underline{n}_{e}^{*}\right)\right)\right]+H\left(\lambda^{\underline{n}_{e}^{*}}-1\right)=0
\end{gathered}
$$

where $H=\frac{c}{2 \theta-1}$ and $\lambda=\frac{1-\theta}{\theta}<1$. Where it must be always the case that $\underline{n}_{e}^{*}<0$ and $\bar{n}_{e}^{*}>0{ }^{39}$ It is also immediate to verify that for $x_{e}=\frac{1}{2}$ it must be the case that $\bar{n}_{e}^{*}=\left|\underline{n}_{e}^{*}\right|$. Notice that the optimal stopping rule $\bar{n}_{e}^{*}$ and $\underline{n}_{e}^{*}$ do not depend on $n$. That is the optimal stopping rule do not change depending on the realization of the signals. ${ }^{40}$ Let's consider the two first order conditions and let's denote them as $f$ and $g$. That is:

$$
\begin{gathered}
f=\left.\frac{\partial V_{e}}{\partial \bar{n}_{e}^{*}}\right|_{\bar{n}_{e}^{*}}=0 \\
g=\left.\frac{\partial V_{e}}{\partial \underline{n}_{e}^{*}}\right|_{n=\underline{n}_{e}^{*}}=0
\end{gathered}
$$

that is $\bar{n}_{e}^{*}$ and $\underline{n}_{e}^{*}$ are the solution of the following system of equations:

$$
\left\{\begin{array}{l}
f\left(\bar{n}_{e}^{*}\left(x_{e}, \delta, c\right), \underline{n}_{e}^{*}\left(x_{e}, \delta, c\right), x_{e}, \delta, c\right)=0 \\
g\left(\bar{n}_{e}^{*}\left(x_{e}, \delta, c\right), \underline{n}_{e}^{*}\left(x_{e}, \delta, c\right), x_{e}, \delta, c\right)=0
\end{array}\right.
$$

In order to obtain the comparative statics, it is necessary to derive the differential of these functions. ${ }^{41}$ That is:

$$
\left\{\begin{array}{l}
\frac{\partial f}{\partial \bar{n}_{e}^{*}} d \bar{n}_{e}^{*}+\frac{\partial f}{\partial n_{e}^{*}} d \underline{n}_{e}^{*}+\frac{\partial f}{\partial x_{e}} d x_{e}+\frac{\partial f}{\partial \delta} d \delta+\frac{\partial f}{\partial c} d c=0 \\
\frac{\partial g}{\partial \bar{n}_{e}^{*}} d \bar{n}_{e}^{*}+\frac{\partial g}{\partial \underline{n}_{e}^{*}} d \underline{n}_{e}^{*}+\frac{\partial g}{\partial x_{e}} d x_{e}+\frac{\partial g}{\partial \delta} d \delta+\frac{\partial g}{\partial c} d c=0
\end{array}\right.
$$

Let's focus on the comparative statics with respect to $x_{e}$. That is, $\frac{d \underline{n}_{e}^{*}}{d x_{e}}$ and $\frac{d \bar{n}_{e}^{*}}{d x_{e}}$ must be determined,

\footnotetext{
${ }^{38}$ The online appendix contains an extended proof where these first order conditions are formally derived.

${ }^{39}$ Suppose not. That is $\underline{n}_{e}^{*}>0$. Thus $\mu\left(\underline{n}_{e}^{*}\right)>\mu(n=0)=p$. If $x_{e}>\frac{1}{2}$, this would imply that $\mu\left(\underline{n}_{e}^{*}\right)>\hat{\mu}_{e}$ and thus $\tau_{e, m}\left(\underline{n}_{e}^{*}\right)=R$ which contradicts the definition of $\underline{n}_{e}^{*}$. If $x_{e}<\frac{1}{2}$, then since $n=0<\underline{n}_{e}^{*}$, this implies that $\tau_{e}(n=0)=L$ and thus the voter would never start sampling. A similar proof applies to show that $\bar{n}_{e}^{*}>0$.

${ }^{40} \mathrm{~A}$ detailed formal derivation of the second order conditions, ensuring that $\left(\bar{n}_{e}^{*}, \underline{n}_{e}^{*}\right)$ is a global maximum, is available upon request to the author.

${ }^{41}$ These comparative statics are determined by treating $n$ as a real number. This mathematical abuse is made for technical convenience (for an analogous treatment see Brocas and Carrillo 2009 and Brocas, Carrillo and Palfrey 2011). At the same time, a marginal change in $\bar{n}_{e}^{*}$ and/or $\underline{n}_{e}^{*}$ has a straightforward interpretation. For example, a marginal increase in the threshold required by a citizen-editor to endorse candidate $j$ simply represents a marginal increase in the probability of such a citizen-editor requiring one more signal in favor of $j$ to endorse her.
} 
holding the other parameter constants. Hence, $d \delta=0$ and $d c=0$. Thus:

$$
\frac{d \underline{n}_{e}^{*}}{d x_{e}}=\frac{\left(\frac{\partial g}{\partial \bar{n}_{e}^{*}} \frac{\partial f}{\partial x_{e}}-\frac{\partial g}{\partial x_{e}} \frac{\partial f}{\partial \bar{n}_{e}^{*}}\right)}{\left(\frac{\partial g}{\partial \underline{n}_{e}^{*}} \frac{\partial f}{\partial \bar{n}_{e}^{*}}-\frac{\partial g}{\partial \bar{n}_{e}^{*}} \frac{\partial f}{\partial \underline{n}_{e}^{*}}\right)}
$$

similarly

$$
\frac{d \bar{n}_{e}^{*}}{d x_{e}}=\frac{\left(\frac{\partial g}{\partial \underline{n}_{e}^{*}} \frac{\partial f}{\partial x_{e}}-\frac{\partial f}{\partial \underline{n}_{e}^{*}} \frac{\partial g}{\partial x_{e}}\right)}{\left(\frac{\partial g}{\partial \bar{n}_{e}^{*}} \frac{\partial f}{\partial \underline{n}_{e}^{*}}-\frac{\partial f}{\partial \bar{n}_{e}^{*}} \frac{\partial g}{\partial \underline{n}_{e}^{*}}\right)}
$$

Then, simple calculations yields:

$$
\frac{d \underline{n}_{e}^{*}}{d x_{e}}=-\frac{2 \lambda^{n_{e}^{*}}\left(\lambda^{\bar{n}_{e}^{*}}+1\right)}{H\left(\lambda^{n_{e}^{*}}-\lambda^{\bar{n}_{e}^{*}}\right)\left(\lambda^{\underline{n}_{e}^{*}}+1\right)}<0
$$

and

$$
\frac{d \bar{n}_{e}^{*}}{d x_{e}}=-\frac{2 \lambda^{\bar{n}_{e}^{*}}\left(\lambda^{n_{e}^{*}}+1\right)}{H\left(\lambda^{\bar{n}_{e}^{*}}+1\right)\left(\lambda^{n_{e}^{*}}-\lambda^{\bar{n}_{e}^{*}}\right)}<0
$$

Moreover, $\left|\frac{d \bar{n}_{e}^{*}}{d x_{e}}\right|>\left|\frac{d \underline{n_{e}^{*}}}{d x_{e}}\right|$ if and only if:

$$
\left(\lambda^{\underline{n}_{e}^{*}}-\lambda^{\bar{n}_{e}^{*}}\right)\left(1-\lambda^{\underline{n}_{e}^{*}} \lambda^{\bar{n}_{e}^{*}}\right)<0
$$

thus since

$$
\left(1-\lambda^{n_{e}^{*}} \lambda^{\bar{n}_{e}^{*}}\right)\left\{\begin{array}{l}
>0 \text { for } x_{e}<1 / 2 \\
=0 \text { for } x_{e}=1 / 2 \\
<0 \text { for } x_{e}>1 / 2
\end{array}\right.
$$

the result follows. Let's now focus on the comparative statics with respect to $\delta$. Using the same methodology as the one described above:

$$
\frac{d \underline{n}_{e}^{*}}{d \delta}=-\frac{2 \lambda^{n_{e}^{*}}\left(1-\lambda^{\bar{n}_{e}^{*}}\right)}{H\left(\lambda^{n_{e}^{*}}+1\right)\left(\lambda^{n_{e}^{*}}-\lambda^{\bar{n}_{e}^{*}}\right)}<0
$$

and

$$
\frac{d \bar{n}_{e}^{*}}{d \delta}=\frac{2 \lambda^{\bar{n}_{e}^{*}}\left(\lambda^{n_{e}^{*}}-1\right)}{H\left(\lambda^{\bar{n}_{e}^{*}}+1\right)\left(\lambda^{n_{e}^{*}}-\lambda^{\bar{n}_{e}^{*}}\right)}>0
$$

Moreover, $\left|\frac{d \bar{n}_{e}^{*}}{d \delta}\right|>\left|\frac{d n_{e}^{*}}{d \delta}\right|$ if and only if:

$$
\left(\lambda^{\bar{n}_{e}^{*}}+\lambda^{\underline{n}_{e}^{*}}\right)\left(\lambda^{\underline{n}_{e}^{*}} \lambda^{\bar{n}_{e}^{*}}-1\right)>0
$$

hence given (20) the results follow. Finally, the comparative statics with respect to $c$ are:

$$
\frac{d \underline{n}_{e}^{*}}{d c}=-\frac{\left(\lambda \underline{n}_{e}^{*}-1\right)}{c(\ln \lambda)\left(\lambda \underline{\underline{n}}_{e}^{*}+1\right)}+\frac{\lambda^{\underline{n}_{e}^{*}}\left(\bar{n}_{e}^{*}-\underline{n}_{e}^{*}\right)\left(1-\lambda^{\bar{n}_{e}^{*}}\right)}{c\left(\lambda_{\underline{n}_{e}^{*}}^{*} \lambda^{\bar{n}}\right)\left(\lambda \underline{n}_{e}^{*}+1\right)}>0
$$

and

$$
\frac{d \bar{n}_{e}^{*}}{d c}=\frac{1-\lambda^{\bar{n}_{e}^{*}}}{c(\ln \lambda)\left(\lambda^{\bar{n}_{e}^{*}}+1\right)}-\frac{\lambda^{\bar{n}_{e}^{*}}\left(\bar{n}_{e}^{*}-\underline{n}_{e}^{*}\right)\left(\lambda^{\underline{n}_{e}^{*}}-1\right)}{\left(\lambda^{\bar{n}_{e}^{*}}+1\right)\left(\lambda^{\underline{n}_{e}^{*}}-\lambda^{\bar{n}_{e}^{*}}\right) c}<0
$$

Q.E.D. 


\section{Proof of Corollary 1}

I first prove how the expected number of signals collected by an editor vary with the editor's ideological preferences and with the parameters of the model. Then, I turn to the analysis of the expected probability of endorsing the low-valence candidate.

The expected number of signals collected by an editor before endorsing a candidate is:

$$
E\left(\left|n_{e}\right|\right)=\operatorname{Pr}\left(n_{e}=\underline{n}_{e}^{*}\right) \cdot\left|\underline{n}_{e}^{*}\right|+\operatorname{Pr}\left(n_{e}=\bar{n}_{e}^{*}\right) \cdot \bar{n}_{e}^{*}
$$

that is:

$$
E\left(\left|n_{e}\right|\right)=\frac{\bar{n}_{e}^{*}\left(\lambda^{n_{e}^{*}}-1\right)\left(\lambda^{\bar{n}_{e}^{*}}+1\right)-\underline{n}_{e}^{*}\left(\lambda^{\underline{n}_{e}^{*}}+1\right)\left(1-\lambda^{\bar{n}_{e}^{*}}\right)}{2\left(\lambda^{\underline{n}_{e}^{*}}-\lambda^{\bar{n}_{e}^{*}}\right)}
$$

Clearly,

$$
\frac{d E\left(\left|n_{e}\right|\right)}{d x_{e}}=\frac{\partial E\left(\left|n_{e}\right|\right)}{\partial \bar{n}_{e}^{*}} \frac{d \bar{n}_{e}^{*}}{d x_{e}}+\frac{\partial E\left(\left|n_{e}\right|\right)}{\partial \underline{n}_{e}^{*}} \frac{d \underline{n}_{e}^{*}}{d x_{e}}
$$

where

$$
\frac{\partial E\left(\left|n_{e}\right|\right)}{\partial \bar{n}_{e}^{*}}=\frac{\left(\lambda^{\underline{n}_{e}^{*}}-1\right)}{2\left(\lambda^{\underline{n}_{e}^{*}}-\lambda^{\bar{n}_{e}^{*}}\right)}\left((\ln \lambda) \lambda^{\bar{n}_{e}^{*}} \frac{\lambda^{\underline{n}_{e}^{*}}+1}{\lambda^{\underline{n}_{e}^{*}}-\lambda^{\bar{n}_{e}^{*}}}\left(\underline{n}_{e}^{*}+\bar{n}_{e}^{*}\right)+\left(\lambda^{\bar{n}_{e}^{*}}+1\right)\right)
$$

and

$$
\frac{\partial E\left(\left|n_{e}\right|\right)}{\partial \underline{n}_{e}^{*}}=\frac{\left(1-\lambda^{\bar{n}_{e}^{*}}\right)}{2\left(\lambda^{n_{e}^{*}}-\lambda^{\bar{n}_{e}^{*}}\right)}\left((\ln \lambda) \lambda^{\underline{n}_{e}^{*}} \frac{\underline{n}_{e}^{*}+\bar{n}_{e}^{*}}{\lambda^{\underline{n}_{e}^{*}}-\lambda^{\bar{n}_{e}^{*}}}\left(1+\lambda^{\bar{n}_{e}^{*}}\right)-\left(\lambda^{\underline{\underline{n}}_{e}^{*}}+1\right)\right)
$$

thus given (18) and (19) derived in the proof of Proposition 1, the sign of $\frac{d E\left(\left|n_{e}\right|\right)}{d x_{e}}$ will be equivalent to the one of the following expression:

$E_{x_{e}}=\left(1-\lambda^{\bar{n}_{e}^{*}} \lambda^{\underline{n}_{e}^{*}}\right)\left(\lambda^{\underline{n}_{e}^{*}}+\lambda^{\bar{n}_{e}^{*}}\right)-\frac{(\ln \lambda)\left(\underline{n}_{e}^{*}+\bar{n}_{e}^{*}\right)}{\lambda^{n_{e}^{*}}-\lambda^{\bar{n}_{e}^{*}}}\left(\frac{\lambda^{2 \bar{n}_{e}^{*}}\left(\lambda^{\underline{n}_{e}^{*}}+1\right)\left(\lambda^{2 \underline{n}_{e}^{*}}-1\right)}{\left(\lambda^{\bar{n}_{e}^{*}}+1\right)}+\frac{\lambda^{2} \underline{n}_{e}^{*}\left(\lambda^{\bar{n}_{e}^{*}}+1\right)\left(1-\lambda^{2 \bar{n}_{e}^{*}}\right)}{\left(\lambda^{n} \underline{n}_{e}^{*}+1\right)}\right)$

Thus, given (20) and given that:

$$
\left(\underline{n}_{e}^{*}+\bar{n}_{e}^{*}\right)\left\{\begin{array}{l}
>0 \text { for } x_{e}<1 / 2 \\
=0 \text { for } x_{e}=1 / 2 \\
<0 \text { for } x_{e}>1 / 2
\end{array}\right.
$$

then:

$$
\frac{d E\left(\left|n_{e}\right|\right)}{d x_{e}}\left\{\begin{array}{l}
>0 \text { for } x_{e}<1 / 2 \\
=0 \text { for } x_{e}=1 / 2 \\
<0 \text { for } x_{e}>1 / 2
\end{array}\right.
$$

Let's now focus on $\delta$. Since

$$
\frac{d E\left(\left|n_{e}\right|\right)}{d \delta}=\frac{\partial E\left(\left|n_{e}\right|\right)}{\partial \bar{n}_{e}^{*}} \frac{d \bar{n}_{e}^{*}}{d \delta}+\frac{\partial E\left(\left|n_{e}\right|\right)}{\partial \underline{n}_{e}^{*}} \frac{d \underline{n}_{e}^{*}}{d \delta}
$$

and given (21) and (22) derived in the proof of Proposition 1, the sign of $\frac{d E\left(\left|n_{e}\right|\right)}{d \delta}$ will be equivalent to the one of the following expression:

$$
\begin{aligned}
E_{\delta} & =\frac{\lambda^{\bar{n}_{e}^{*}}\left(\lambda^{\bar{n}_{e}^{*}}+1\right)\left(\lambda^{\underline{n}_{e}^{*}}-1\right)^{2}}{\left(\lambda^{\bar{n}_{e}^{*}}+1\right)}+\frac{\lambda_{\underline{n}_{e}^{*}}\left(\lambda^{\underline{n}_{e}^{*}}+1\right)\left(1-\lambda^{\bar{n}_{e}^{*}}\right)^{2}}{\left(\lambda \underline{\underline{n}}_{e}^{*}+1\right)} \\
- & \frac{(\ln \lambda)\left(\underline{n}_{e}^{*}+\bar{n}_{e}^{*}\right)\left(1-\lambda \underline{\underline{n}}_{e}^{*} \lambda^{\bar{n}_{e}^{*}}\right)\left(1+\lambda^{\underline{n}_{e}^{*}} \lambda^{\bar{n}_{e}^{*}}\right)\left(\lambda^{2 \underline{n}_{e}^{*}}-\lambda^{2 \bar{n}_{e}^{*}}\right)}{\left(\lambda^{n_{e}^{*}}-\lambda^{\bar{n}_{e}^{*}}\right)\left(\lambda^{\underline{n}_{e}^{*}}+1\right)\left(\lambda^{\bar{n}_{e}^{*}}+1\right)}
\end{aligned}
$$

where the above expression is always positive since $\left(\underline{n}_{e}^{*}+\bar{n}_{e}^{*}\right)$ and $\left(1-\lambda \underline{n}_{e}^{*} \lambda^{\bar{n}_{e}^{*}}\right)$ are both positive for $x_{e}<1 / 2$ and both negative for $x_{e}>1 / 2$. Moreover, for $x_{e}=1 / 2$ the above expression reduces 
to $\frac{2\left[1-\lambda\left(\left.\bar{n}_{e}^{*}\right|_{x_{e}=1 / 2}\right)\right]^{2}}{{ }_{\lambda}\left(\left.\bar{n}_{e}^{*}\right|_{x_{e}=1 / 2}\right)}>0$.

Let's now focus on $c$. Since

$$
\frac{d E\left(\left|n_{e}\right|\right)}{d c}=\frac{\partial E\left(\left|n_{e}\right|\right)}{\partial c} \frac{d \bar{n}_{e}^{*}}{d c}+\frac{\partial E\left(\left|n_{e}\right|\right)}{\partial \underline{n}_{e}^{*}} \frac{d \underline{n}_{e}^{*}}{d c}
$$

and given (23) and (24) derived in the proof of Proposition 1, the sign of $\frac{d E\left(\left|n_{e}\right|\right)}{d c}$ will be equivalent to the one of the following expression:

$$
\begin{aligned}
E_{c} & =\frac{\left(\lambda^{\underline{n}_{e}^{*}}-1\right)\left(2-\lambda^{\bar{n}_{e}^{*}}\right)}{c \ln \lambda}-\frac{\left(\bar{n}_{e}^{*}-\underline{n}_{e}^{*}\right)\left(\lambda^{\underline{n}_{e}^{*}}\left(1-\lambda^{\bar{n}_{e}^{*}}\right)+\lambda^{\bar{n}_{e}^{*}}\left(\lambda^{\underline{n}_{e}^{*}}-1\right)^{2}\right)}{c\left(\lambda^{n_{e}^{*}}-\lambda^{\bar{n}_{e}^{*}}\right)} \\
& +\frac{\left(\underline{n}_{e}^{*}+\bar{n}_{e}^{*}\right)\left(\lambda^{\underline{n}_{e}^{*}} \lambda^{\bar{n}_{e}^{*}}-1\right)\left(\lambda^{\underline{n}_{e}^{*}}-1\right)\left(1-\lambda^{\bar{n}_{e}^{*}}\right)}{c\left(\lambda^{n_{e}^{*}}+1\right)\left(\lambda^{\bar{n}_{e}^{*}}+1\right)}-\frac{(\ln \lambda)\left(\underline{n}_{e}^{*}+\bar{n}_{e}^{*}\right)\left(\lambda^{2 \underline{n}_{e}^{*}} \lambda^{2 \bar{n}_{e}^{*}}-1\right)\left(\bar{n}_{e}^{*}-\underline{n}_{e}^{*}\right)\left(\lambda^{2 \underline{n}_{e}^{*}}-\lambda^{2 \bar{n}_{e}^{*}}\right)}{c\left(\lambda \underline{n}_{e}^{*}-\lambda^{\bar{n}_{e}^{*}}\right)^{2}\left(\lambda^{n_{e}^{*}}+1\right)\left(\lambda^{\bar{n}_{e}^{*}}+1\right)}
\end{aligned}
$$

where the above is always negative. Indeed the first two terms are clearly negative and the second and third negative are always negative since $\left(\underline{n}_{e}^{*}+\bar{n}_{e}^{*}\right)$ and $\left(\lambda_{e}^{n_{e}^{*}} \lambda^{\bar{n}_{e}^{*}}-1\right)$ have always opposite signs for $x_{e}<1 / 2$ or $x_{e}>1 / 2$ (similarly, $\left(\underline{n}_{e}^{*}+\bar{n}_{e}^{*}\right)$ and $\left(\lambda^{2} \underline{n}_{e}^{*} \lambda^{2 \bar{n}_{e}^{*}}-1\right)$ have also always opposite signs for $x_{e}<1 / 2$ or $\left.x_{e}>1 / 2\right)$. Moreover, for $x_{e}=1 / 2$ the above expression reduces to:

$$
\frac{\left(2-\lambda^{\left(\left.\bar{n}_{e}^{*}\right|_{x_{e}=1 / 2}\right)}\right)\left[\left(1-\lambda^{2\left(\left.\bar{n}_{e}^{*}\right|_{x_{e}=1 / 2}\right)}\right)-2(\ln \lambda)\left(\left.\bar{n}_{e}^{*}\right|_{x_{e}=1 / 2}\right) \lambda^{\left(\left.\bar{n}_{e}^{*}\right|_{x_{e}=1 / 2}\right)}\right]}{c(\ln \lambda) \lambda^{\left(\left.\bar{n}_{e}^{*}\right|_{x_{e}=1 / 2}\right)}\left(1+\lambda^{\left(\left.\bar{n}_{e}^{*}\right|_{x_{e}=1 / 2}\right)}\right)}<0
$$

This proves the first part of the corollary relative to the comparative statics on the expected number of signals collected by an editor before endorsing a candidate. Let's now analyze the probability of an editor endorsing the low-quality candidate. Since:

$$
\operatorname{Pr}\left(\tau_{e}=L \mid s=r\right)=\frac{2 \mu\left(\bar{n}_{e}^{*}\right)-1}{\mu\left(\bar{n}_{e}^{*}\right)-\mu\left(\underline{n}_{e}^{*}\right)} \mu\left(\underline{n}_{e}^{*}\right)
$$

and

$$
\operatorname{Pr}\left(\tau_{e}=R \mid s=l\right)=\frac{1-2 \mu\left(\underline{n}_{e}^{*}\right)}{\mu\left(\bar{n}_{e}^{*}\right)-\mu\left(\underline{n}_{e}^{*}\right)}\left[1-\mu\left(\bar{n}_{e}^{*}\right)\right]
$$

Thus it is easy to verify that $\operatorname{Pr}\left(\tau_{e}=L \mid s=r\right)$ is decreasing in $x_{e}$ and $\operatorname{Pr}\left(\tau_{e}=R \mid s=l\right)$ is increasing in $x_{e}$. Moreover, the ex-ante probability of making a wrong choice is:

$$
\operatorname{Pr}(\text { error })=\operatorname{Pr}(s=r) \operatorname{Pr}\left(\tau_{e}=L \mid s=r\right)+\operatorname{Pr}(s=l) \operatorname{Pr}\left(\tau_{e}=R \mid s=l\right)
$$

hence:

$$
\operatorname{Pr}(\text { error })=\frac{\lambda^{\bar{n}_{e}^{*}}\left(\lambda \underline{n}_{e}^{*}-1\right)+\left(1-\lambda^{\bar{n}_{e}^{*}}\right)}{2\left(\lambda \underline{\underline{n}}_{e}^{*}-\lambda^{\bar{n}_{e}^{*}}\right)}
$$

It is now possible to perform the comparative statics upon this probability. First of all:

$$
\begin{gathered}
\frac{\partial \operatorname{Pr}(\text { error })}{\partial \bar{n}_{e}^{*}}=\frac{1}{2}(\ln \lambda) \lambda^{\bar{n}_{e}^{*}} \frac{\left(\lambda \underline{n}_{e}^{*}-1\right)^{2}}{\left(\lambda \underline{n}_{e}^{*}-\lambda^{\bar{n}_{e}^{*}}\right)^{2}}<0 \\
\frac{\partial \operatorname{Pr}(\text { error })}{\partial \underline{n}_{e}^{*}}=-\frac{1}{2}(\ln \lambda) \lambda^{\underline{n}_{e}^{*}} \frac{\left(1-\lambda^{\bar{n}_{e}^{*}}\right)^{2}}{\left(\lambda^{n_{e}^{*}}-\lambda^{\bar{n}_{e}^{*}}\right)^{2}}>0
\end{gathered}
$$

Hence, since $\frac{d \bar{n}_{e}^{*}}{d c}<0$ and $\frac{d \underline{n}_{e}^{*}}{d c}>0$, then $\frac{d \operatorname{Pr}(e r r o r)}{d c}>0$. Similarly, since $\frac{d \bar{n}_{e}^{*}}{d \delta}>0$ and $\frac{d n_{e}^{*}}{d \delta}<0$, then $\frac{d \operatorname{Pr}(\text { error })}{d \delta}<0$. 
Finally given (18) and (19) derived in the proof of Proposition $1, \frac{d \operatorname{Pr}(\text { error })}{d x_{e}}>0$ if and only if:

$$
\frac{\left(\lambda^{2 \underline{n}_{e}^{*}}-\lambda^{2 \bar{n}_{e}^{*}}\right)\left(1+\lambda^{\underline{n}_{e}^{*}} \lambda^{\bar{n}_{e}^{*}}\right)\left(1-\lambda^{\underline{n}_{e}^{*}} \lambda^{\bar{n}_{e}^{*}}\right)}{\left(\lambda \underline{\underline{n}}_{e}^{*}+1\right)\left(\lambda^{\bar{n}_{e}^{*}}+1\right)}<0
$$

Thus, given (20):

$$
\frac{d \operatorname{Pr}(\text { error })}{d x_{e}}\left\{\begin{array}{l}
<0 \text { for } x<\frac{1}{2} \\
=0 \text { for } x=\frac{1}{2} \\
>0 \text { for } x>\frac{1}{2}
\end{array}\right.
$$

\section{Q.E.D.}

\section{Proof of Lemma 1}

It is immediate to verify that $\left(\tilde{x}_{e}-\hat{x}_{e}\right)$ is decreasing in $C$. Let's now focus on $\tilde{x}_{e}$. Then:

$$
\begin{aligned}
& \frac{d \tilde{x}_{e}\left(\bar{n}_{e}^{*}, \underline{n}_{e}^{*}\right)}{d \bar{n}_{e}^{*}}=-C(\ln \lambda) \frac{\lambda^{\bar{n}_{e}^{*}}\left(\lambda \underline{n}_{e}^{*}-1\right)}{\left(\lambda_{\underline{n}_{e}^{*}}^{*}+1\right)\left(1-\lambda^{\bar{n}_{e}^{*}}\right)^{2}}>0 \\
& \frac{d \tilde{x}_{e}\left(\bar{n}_{e}^{*}, \underline{n}_{e}^{*}\right)}{d \underline{n}_{e}^{*}}=(\ln \lambda) \frac{\lambda_{\underline{n}_{e}^{*}}}{\left(\lambda \underline{n}_{e}^{*}+1\right)^{2}}\left(2 \delta-C \frac{\left(\lambda^{\bar{n}_{e}^{*}}+1\right)}{\left(1-\lambda^{\bar{n}_{e}^{*}}\right)}\right)<0
\end{aligned}
$$

The it is immediate to verify that $\tilde{x}_{e}$ is increasing in $\delta$. Let's now analyze how $\tilde{x}_{e}$ changes as $x_{e}$ increases. First, I want to prove that for any $x_{e}<1 / 2$ it is always the case that $d \tilde{x}_{e} / d x_{e}>0$. From the proof of Proposition 1 we know that for $x_{e}<1 / 2,\left|\frac{d \underline{n}_{e}^{*}}{d x_{e}}\right|>\left|\frac{d \bar{n}_{e}^{*}}{d x_{e}}\right|$. Hence, a sufficient condition to ensure that $d \tilde{x}_{e} / d x_{e}>0$ is simply:

$$
\left|\frac{d \tilde{x}_{e}\left(\bar{n}_{e}^{*}, \underline{n}_{e}^{*}\right)}{d \underline{n}_{e}^{*}}\right|>\left|\frac{d \tilde{x}_{e}\left(\bar{n}_{e}^{*}, \underline{n}_{e}^{*}\right)}{d \bar{n}_{e}^{*}}\right|
$$

which is true if and only if:

$$
C\left(\frac{\lambda^{\bar{n}_{e}^{*}}\left(\lambda^{2 \underline{n}_{e}^{*}}-1\right)}{\lambda^{n_{e}^{*}}\left(1-\lambda^{\bar{n}_{e}^{*}}\right)^{2}}+\frac{\left(\lambda^{\bar{n}_{e}^{*}}+1\right)}{\left(1-\lambda^{\bar{n}_{e}^{*}}\right)}\right)<2 \delta
$$

Since $\frac{\partial}{\partial \bar{n}_{e}^{*}}\left(\frac{1-\lambda \bar{n}_{e}^{*}}{1+\bar{n}_{e}^{*}}\right)>0$ and $\frac{d \bar{n}_{e}^{*}}{d x_{e}}<0$, then $\delta\left(\frac{1-\lambda^{\bar{n}_{e}^{*}}}{1+\lambda^{\bar{n}_{e}^{*}}}\right) \geq C^{\max }$. Hence, a sufficient condition for the above condition to be always true is:

$$
\left(\lambda^{\bar{n}_{e}^{*}} \lambda^{\underline{n}_{e}^{*}}-1\right)\left(\lambda^{\bar{n}_{e}^{*}}+\lambda^{\underline{n_{e}^{*}}}\right)<0
$$

which it is always the case for $x_{e}<1 / 2$. Moreover, for $x_{e}=1 / 2, \underline{n}_{e}^{*}=-\bar{n}_{e}^{*}$ and thus:

$$
\left.\frac{d \tilde{x}_{e}}{d x_{e}}\right|_{x_{e}=1 / 2}=-\frac{4}{H}(\ln \lambda) \frac{\lambda^{2 \bar{n}_{e}^{*}}\left(\delta\left(1-\lambda^{\bar{n}_{e}^{*}}\right)-C\left(\lambda^{\bar{n}_{e}^{*}}+1\right)\right)}{\left(1-\lambda^{2 \bar{n}_{e}^{*}}\right)\left(\left(1-\lambda^{3 \bar{n}_{e}^{*}}\right)+\lambda^{\bar{n}_{e}^{*}}\left(1-\lambda^{\bar{n}_{e}^{*}}\right)\right)}>0
$$

Hence, for any $x_{e} \leq 1 / 2$, it is always the case that $d \tilde{x}_{e} / d x_{e}>0$. Let's analyze now the case where $x_{e}>1 / 2$. Then, $d \tilde{x}_{e} / d x_{e}>0$ if and only if:

$$
C<\tilde{C} \equiv 2 \delta \frac{\lambda^{2 \underline{n}_{e}^{*}}\left(1-\lambda^{2 \bar{n}_{e}^{*}}\right)^{2}}{\lambda^{2 \bar{n}_{e}^{*}}\left(\lambda^{2} \underline{n}_{e}^{*}-1\right)\left(\lambda^{n_{e}^{*}}+1\right)^{2}+\lambda^{2 \underline{n}_{e}^{*}}\left(\lambda^{\bar{n}_{e}^{*}}+1\right)^{2}\left(1-\lambda^{2 \bar{n}_{e}^{*}}\right)}
$$

hence $\tilde{C}>0$. Let's now analyze how $\tilde{C}$ changes when $x_{e}$ increases:

$$
\frac{\partial \tilde{C}}{\partial \bar{n}_{e}^{*}}=-\frac{4 \delta(\ln \lambda)\left(1-\lambda^{2 \bar{n}_{e}^{*}}\right) \lambda^{2 \underline{n}_{e}^{*}+\bar{n}_{e}^{*}}}{\lambda^{2 \bar{n}_{e}^{*}}\left(\lambda^{\underline{n}_{e}^{*}}+1\right)^{2}\left(\lambda^{2 \underline{n}_{e}^{*}}-1\right)+\lambda^{2 \underline{n}_{e}^{*}}\left(\lambda^{\bar{n}_{e}^{*}}+1\right)^{2}\left(1-\lambda^{2 \bar{n}_{e}^{*}}\right)} Y>0
$$


where

$Y=\left(2 \lambda^{\bar{n}_{e}^{*}}+\frac{\left(1-\lambda^{2 \bar{n}_{e}^{*}}\right)\left[\left(\lambda^{2 \underline{n}_{e}^{*}}\left(\lambda^{\bar{n}_{e}^{*}}+1\right)\left(1-\lambda^{2 \bar{n}_{e}^{*}}\right)-\left(\lambda^{\bar{n}_{e}^{*}}+1\right)^{2} \lambda^{2 \underline{n}_{e}^{*}+\bar{n}_{e}^{*}}+\lambda^{\bar{n}_{e}^{*}}\left(\lambda^{\underline{n}_{e}^{*}}+1\right)^{2}\left(\lambda^{2 \underline{n}_{e}^{*}}-1\right)\right)\right]}{\left(\lambda^{2 \bar{n}_{e}^{*}}\left(\lambda^{n_{e}^{*}}+1\right)^{2}\left(\lambda^{2 \underline{n}_{e}^{*}}-1\right)+\lambda^{2 \underline{n}_{e}^{*}}\left(\lambda^{\bar{n}_{e}^{*}}+1\right)^{2}\left(1-\lambda^{2 \bar{n}_{e}^{*}}\right)\right)}\right)>0$

since $\lambda^{\bar{n}_{e}^{*}}\left(\lambda^{n_{e}^{*}}+1\right)^{2}\left(\lambda^{2 \underline{n}_{e}^{*}}-1\right)>\left(\lambda^{\bar{n}_{e}^{*}}+1\right)^{2} \lambda^{2 \underline{n}_{e}^{*}+\bar{n}_{e}^{*}}>0$. Moreover:

$$
\frac{\partial \tilde{C}}{\partial \underline{n}_{e}^{*}}=-\frac{4 \delta(\ln \lambda) \lambda^{2} \underline{n}_{e}^{*}\left(\lambda^{\bar{n}_{e}^{*}}-1\right)^{2}\left(\lambda^{\bar{n}_{e}^{*}}+1\right)^{2}\left(\lambda^{\underline{n}_{e}^{*}}+\lambda^{4 \underline{n}_{e}^{*}}+\lambda^{3 \underline{n}_{e}^{*}}+1\right)\left(\lambda^{2 \bar{n}_{e}^{*}}\right)}{\left(\lambda^{2 \bar{n}_{e}^{*}}\left(\lambda^{\underline{n}_{e}^{*}}+1\right)^{2}\left(\lambda^{2 \underline{n}_{e}^{*}}-1\right)+\lambda^{2 \underline{n}_{e}^{*}}\left(\lambda^{\bar{n}_{e}^{*}}+1\right)^{2}\left(1-\lambda^{2 \bar{n}_{e}^{*}}\right)\right)^{2}}>0
$$

hence since $\frac{d \bar{n}_{e}^{*}}{d x_{e}}<0$ and $\frac{d \underline{n}_{e}^{*}}{d x_{e}}<0$ :

$$
\frac{d \tilde{C}}{d x_{e}}=\frac{\partial \tilde{C}}{\partial \bar{n}_{e}^{*}} \frac{d \bar{n}_{e}^{*}}{d x_{e}}+\frac{\partial \tilde{C}}{\partial \underline{n}_{e}^{*}} \frac{d \underline{n}_{e}^{*}}{d x_{e}}<0
$$

Hence, $\tilde{x}_{e}$ will be increasing in $x_{e}$ for $x_{e}>1 / 2$ if and only if $C<\tilde{C}$. That is, since $\frac{d \tilde{C}}{d x_{e}}<0, \tilde{x}_{e}$ will be increasing in $x_{e}$ only as long as $x_{e}<x_{e_{R}}^{\max }$, where:

$$
\tilde{C}\left(\bar{n}_{e}^{*}\left(x_{e_{R}}^{\max }\right), \underline{n}_{e}^{*}\left(x_{e_{R}}^{\max }\right)\right)=C
$$

Moreover, since $\frac{d \tilde{C}}{d x_{e}}<0, \tilde{C}^{\max }<\lim _{x_{e} \rightarrow 1 / 2} \tilde{C}=C^{\max }$. Finally, since $\delta \in\left(0, \frac{1}{2}\right], x_{e_{R}}^{\max }<1$. Specifically, for $\delta<1 / 2$ an editor with preferences $x_{e_{R}}=1$ would never endorse a leftist candidate since, trivially, $\hat{\mu}_{\left(x_{e_{R}}=1\right)}=0$ (i.e., $\bar{n}_{e}^{*}\left(x_{e_{R}}=1\right)=0$ ) which implies that $\tilde{C}\left(x_{e_{R}}=1\right)=0$. For $\delta=1 / 2$, an editor with preferences $x_{e_{R}}=1$ will endorse a leftist candidate if and only if $\mu(n)=0$. That is, if and only if $n=-\infty$. Hence, necessary conditions for this to be verified are $\underline{n}_{e}\left(x_{e_{R}}=1\right) \rightarrow-\infty$ and $\bar{n}_{e}\left(x_{e_{R}}=1\right) \rightarrow 0$. As shown by Proposition 1 , for $x_{e}>\frac{1}{2}$ it is the case that $\left|\frac{d \bar{n}_{e}^{*}}{d x_{e}}\right|>\left|\frac{d \underline{n}_{e}^{*}}{d x_{e}}\right|$. That is, when $x_{e_{R}} \rightarrow 1$ it must be the case that $\bar{n}_{e_{R}}^{*} \rightarrow 0$ but $\bar{n}_{e_{R}}^{*}-\left|\underline{n}_{e_{R}}^{*}\right| \nrightarrow-\infty$. In turn, this implies that $\tilde{C} \rightarrow 0$ when $x_{e_{R}} \rightarrow 1$.

Let's now focus on $\hat{x}_{e}$. Then:

$$
\begin{aligned}
\frac{d \hat{x}_{e}}{d \bar{n}_{e}^{*}} & =(\ln \lambda) \frac{\lambda^{\bar{n}_{e}^{*}}\left(2 \delta\left(\lambda^{\underline{n}_{e}^{*}}-1\right)-C\left(\lambda^{\underline{n}_{e}^{*}}+1\right)\right)}{\left(\lambda^{n_{e}^{*}}-1\right)\left(\lambda^{\bar{n}_{e}^{*}}+1\right)^{2}}<0 \\
\frac{d \hat{x}_{e}}{d \underline{n}_{e}^{*}} & =-C(\ln \lambda) \lambda^{\underline{n}_{e}^{*}} \frac{1-\lambda^{\bar{n}_{e}^{*}}}{\left(\lambda^{\bar{n}_{e}^{*}}+1\right)\left(\lambda^{\underline{n}_{e}^{*}}-1\right)^{2}}>0
\end{aligned}
$$

Thus it is immediate to verify that $\hat{x}_{e}$ is decreasing in $\delta$. Let's now analyze how $\hat{x}_{e}$ changes as $x_{e}$ increases. First, I want to prove that for any $x_{e}>1 / 2$ it is always the case that $d \hat{x}_{e} / d x_{e}>0$. As shown in the proof of Proposition 1, for $x_{e}>1 / 2$, then $\left|\frac{d \underline{n}_{e}^{*}}{d x_{e}}\right|<\left|\frac{d \bar{n}_{e}^{*}}{d x_{e}}\right|$. Hence, a sufficient condition to ensure that $d \hat{x}_{e} / d x_{e}>0$ is simply:

$$
\left|\frac{d \hat{x}_{e}\left(\bar{n}_{e}^{*}, \underline{n}_{e}^{*}\right)}{d \underline{n}_{e}^{*}}\right|<\left|\frac{d \hat{x}_{e}\left(\bar{n}_{e}^{*}, \underline{n}_{e}^{*}\right)}{d \bar{n}_{e}^{*}}\right|
$$

that is

$$
C\left(\frac{\lambda^{\underline{n}_{e}^{*}}\left(1-\lambda^{2 \bar{n}_{e}^{*}}\right)}{\lambda^{\bar{n}_{e}^{*}}\left(\lambda^{n_{e}^{*}}-1\right)^{2}}+\frac{\left(\lambda^{n_{e}^{*}}+1\right)}{\left(\lambda^{n_{e}^{*}}-1\right)}\right)<2 \delta
$$

moreover since $C^{\max } \leq \frac{1-\lambda_{\bar{n}_{e}^{*}}^{*}}{\left(\lambda^{\bar{n}_{e}^{*}}+1\right)} \delta<\delta \frac{\lambda_{n_{e}^{*}}^{n_{e}^{*}}}{\left(\lambda_{e}^{n_{e}^{*}}+1\right)}$, a sufficient condition for the above to be verified 
becomes:

$$
\frac{\left(1-\lambda^{\bar{n}_{e}^{*}} \lambda^{\underline{n}_{e}^{*}}\right)\left(\lambda^{\bar{n}_{e}^{*}}+\lambda^{\underline{n}_{e}^{*}}\right)}{\lambda^{\bar{n}_{e}^{*}}\left(\lambda^{2} \underline{n}_{e}^{*}-1\right)}<0
$$

hence since $\left(1-\lambda^{\bar{n}_{e}^{*}} \lambda \underline{\underline{n}}_{e}^{*}\right)<0$ for $x_{e}>1 / 2$, we have proved that for $x_{e}>1 / 2$ it is always the case that $d \hat{x}_{e} / d x_{e}>0$. Moreover, for $x_{e}=1 / 2, \underline{n}_{e}^{*}=-\bar{n}_{e}^{*}$ and thus:

$$
\left.\frac{d \hat{x}_{e}}{d x_{e}}\right|_{x_{e}=1 / 2}=-\frac{4}{H}(\ln \lambda) \frac{\lambda^{2 \bar{n}_{e}^{*}}\left(\delta\left(1-\lambda^{\bar{n}_{e}^{*}}\right)-C\left(\lambda^{\bar{n}_{e}^{*}}+1\right)\right)}{\left(1-\lambda^{2 \bar{n}_{e}^{*}}\right)\left(\left(1-\lambda^{3 \bar{n}_{e}^{*}}\right)+\lambda^{\bar{n}_{e}^{*}}\left(1-\lambda^{\bar{n}_{e}^{*}}\right)\right)}>0
$$

Hence, for any $x_{e} \geq 1 / 2$, it is always the case that $d \hat{x}_{e} / d x_{e}>0$. Let's now analyze the case where $x_{e}<1 / 2$. In this case, $d \hat{x}_{e} / d x_{e}>0$ if and only if:

$$
C<\hat{C} \equiv 2 \delta \frac{\lambda^{2 \bar{n}_{e}^{*}}\left(\lambda^{2 \underline{n}_{e}^{*}}-1\right)^{2}}{\lambda^{2 \underline{n}_{e}^{*}}\left(1-\lambda^{2 \bar{n}_{e}^{*}}\right)\left(\lambda^{\bar{n}_{e}^{*}}+1\right)^{2}+\lambda^{2 \bar{n}_{e}^{*}}\left(\lambda^{2 \underline{n}_{e}^{*}}-1\right)\left(\lambda^{\underline{n}_{e}^{*}}+1\right)^{2}}
$$

hence $\hat{C}>0$. Let's now analyze how $\hat{C}$ changes when $x_{e}$ increases. First of all:

$$
\frac{\partial \hat{C}}{\partial \bar{n}_{e}^{*}}=4 \delta(\ln \lambda) \lambda^{2 \bar{n}_{e}^{*}} \frac{\left(\lambda^{2 \underline{n}_{e}^{*}}-1\right)^{2}\left(\lambda^{\bar{n}_{e}^{*}}+\lambda^{4 \bar{n}_{e}^{*}}+\lambda^{3 \bar{n}_{e}^{*}}+1\right)\left(\lambda^{2 \underline{n}_{e}^{*}}\right)}{\left(\lambda^{2 \bar{n}_{e}^{*}}\left(\lambda^{n_{e}^{*}}+1\right)^{2}\left(\lambda^{2 \underline{n}_{e}^{*}}-1\right)+\lambda^{2 \underline{n}_{e}^{*}}\left(\lambda^{\bar{n}_{e}^{*}}+1\right)^{2}\left(1-\lambda^{2 \bar{n}_{e}^{*}}\right)\right)^{2}}<0
$$

and

$$
\frac{\partial \hat{C}}{\partial \underline{n}_{e}^{*}}=\frac{4 \delta(\ln \lambda)\left(\lambda^{2 \underline{n}_{e}^{*}}-1\right) \lambda_{\underline{n}_{e}^{*}+2 \bar{n}_{e}^{*}}}{\left(\lambda^{2 \bar{n}_{e}^{*}}\left(\lambda^{n_{e}^{*}}+1\right)^{2}\left(\lambda^{2} \underline{n}_{e}^{*}-1\right)+\lambda^{2} \underline{n}_{e}^{*}\left(\lambda^{\bar{n}_{e}^{*}}+1\right)^{2}\left(1-\lambda^{2 \bar{n}_{e}^{*}}\right)\right)^{2}} W<0
$$

where

$W=2 \lambda^{\bar{n}_{e}^{*}} \lambda^{\underline{n}_{e}^{*}}\left(\left(\lambda^{2 \underline{n}_{e}^{*}}-\lambda^{2 \bar{n}_{e}^{*}}\right)+\left(1+\lambda^{2 \underline{n}_{e}^{*}} \lambda^{\bar{n}_{e}^{*}}\right)\left(1-\lambda^{\bar{n}_{e}^{*}}\right)\right)+\left(\lambda^{\underline{n}_{e}^{*}}-\lambda^{2 \bar{n}_{e}^{*}}\right)\left(\lambda^{2 \underline{n}_{e}^{*}}+\lambda^{\underline{n}_{e}^{*}} \lambda^{2 \bar{n}_{e}^{*}}+\lambda^{3 \underline{n}_{e}^{*}} \lambda^{2 \bar{n}_{e}^{*}}+1\right)$

hence since $\frac{d \bar{n}_{e}^{*}}{d x_{e}}<0$ and $\frac{d \underline{n}_{e}^{*}}{d x_{e}}<0$ :

$$
\frac{d \hat{C}}{d x_{e}}=\frac{\partial \hat{C}}{\partial \bar{n}_{e}^{*}} \frac{d \bar{n}_{e}^{*}}{d x_{e}}+\frac{\partial \hat{C}}{\partial \underline{n}_{e}^{*}} \frac{d \underline{n}_{e}^{*}}{d x_{e}}>0
$$

Hence, $\hat{x}_{e}$ will be increasing in $x_{e}$ for $x_{e}<1 / 2$ if and only if $C<\hat{C}$. That is, since $\frac{d \hat{C}}{d x_{e}}>0, \tilde{x}_{e}$ will be increasing in $x_{e}$ only as long as $x_{e}>x_{e_{L}}^{\min }$, where $x_{e_{L}}^{\min }$ is such that:

$$
\hat{C}\left(\bar{n}_{e}^{*}\left(x_{e_{L}}^{\min }\right), \underline{n}_{e}^{*}\left(x_{e_{L}}^{\min }\right)\right)=C
$$

Moreover, since $\frac{d \hat{C}}{d x_{e}}>0, \hat{C}^{\max }<\lim _{x_{e} \rightarrow 1 / 2} \hat{C}=C^{\max }$. Finally, by using an analogous proof to the one employed above to show that $x_{e_{R}}^{\max }<1$, it is immediate to see that since $\delta \in\left(0, \frac{1}{2}\right]$, it is always the case that $x_{e_{L}}^{\min }>0$ and that $\hat{C} \rightarrow 0$ when $x_{e_{L}} \rightarrow 0$. Q.E.D.

\section{Proof of Proposition 2}

The optimal strategy for a profit maximizing monopolist media outlet is to choose an editor with idiosyncratic preference $x_{e}$ such that its profits are maximized. That is $x_{e}^{m o n}$ must be such that:

$$
\frac{d \Pi}{d x_{e}}=\frac{d \Pi}{d \bar{n}_{e}^{*}} \frac{d \bar{n}_{e}^{*}}{d x_{e}}+\frac{d \Pi}{d \underline{n}_{e}^{*}} \frac{d \underline{n}_{e}^{*}}{d x_{e}}=0
$$

Where:

$$
\frac{d \Pi}{d \bar{n}_{e}^{*}}=\frac{d F\left(\tilde{x}_{e}\right)}{d \bar{n}_{e}^{*}}-\frac{d F\left(\hat{x}_{e}\right)}{d \bar{n}_{e}^{*}}
$$




$$
\frac{d \Pi}{d \underline{n}_{e}^{*}}=\frac{d F\left(\tilde{x}_{e}\right)}{d \underline{n}_{e}^{*}}-\frac{d F\left(\hat{x}_{e}\right)}{d \underline{n}_{e}^{*}}
$$

where $\frac{d F\left(\tilde{x}_{e}\right)}{d \bar{n}_{e}^{*}}=\frac{d}{d \bar{n}_{e}^{*}} \int_{\delta}^{\tilde{x}_{e}\left(\bar{n}_{e}^{*}\right)} f(x) d x$. Hence applying Leibniz's rule:

$$
\frac{d F\left(\tilde{x}_{e}\right)}{d \bar{n}_{e}^{*}}=\frac{d}{d \bar{n}_{e}^{*}} \int_{\delta}^{\tilde{x}_{e}\left(\bar{n}_{e}^{*}, \underline{n}_{e}^{*}\right)} f(x) d x=f\left(\tilde{x}_{e}\left(\bar{n}_{e}^{*}, \underline{n}_{e}^{*}\right)\right) \frac{d \tilde{x}_{e}\left(\bar{n}_{e}^{*}, \underline{n}_{e}^{*}\right)}{d \bar{n}_{e}^{*}}
$$

thus,

similarly

$$
\frac{d \Pi}{d \bar{n}_{e}^{*}}=f\left(\tilde{x}_{e}\left(\bar{n}_{e}^{*}, \underline{n}_{e}^{*}\right)\right) \frac{d \tilde{x}_{e}\left(\bar{n}_{e}^{*}, \underline{n}_{e}^{*}\right)}{d \bar{n}_{e}^{*}}-f\left(\hat{x}_{e}\left(\bar{n}_{e}^{*}, \underline{n}_{e}^{*}\right)\right) \frac{d \hat{x}_{e}\left(\bar{n}_{e}^{*}\right)}{d \bar{n}_{e}^{*}}
$$

$$
\frac{d \Pi}{d \underline{n}_{e}^{*}}=f\left(\tilde{x}_{e}\left(\bar{n}_{e}^{*}, \underline{n}_{e}^{*}\right)\right) \frac{d \tilde{x}_{e}\left(\bar{n}_{e}^{*}, \underline{n}_{e}^{*}\right)}{d \underline{n}_{e}^{*}}-f\left(\hat{x}_{e}\left(\bar{n}_{e}^{*}, \underline{n}_{e}^{*}\right)\right) \frac{d \hat{x}_{e}\left(\bar{n}_{e}^{*}, \underline{n}_{e}^{*}\right)}{d \underline{n}_{e}^{*}}
$$

Hence the first order condition becomes:

$$
\frac{d \tilde{x}_{e} / d x_{e}}{d \hat{x}_{e} / d x_{e}}=\frac{f\left(\hat{x}_{e}\left(\bar{n}_{e}^{*}, \underline{n}_{e}^{*}\right)\right)}{f\left(\tilde{x}_{e}\left(\bar{n}_{e}^{*}, \underline{n}_{e}^{*}\right)\right)}
$$

where:

$$
\begin{aligned}
& \frac{d \tilde{x}_{e}}{d x_{e}}=\frac{-2(\ln \lambda)}{H\left(\lambda \underline{n}_{e}^{*}-\lambda^{\bar{n}_{e}^{*}}\right)}\left(2 \delta \frac{\lambda^{2 \underline{n}_{e}^{*}}\left(\lambda^{\bar{n}_{e}^{*}}+1\right)}{\left(\lambda \underline{n}_{e}^{*}+1\right)^{3}}-C\left(\frac{\lambda^{2 \bar{n}_{e}^{*}}\left(\lambda^{\underline{n}_{e}^{*}}-1\right)}{\left(\lambda^{\bar{n}_{e}^{*}}+1\right)\left(1-\lambda^{\bar{n}_{e}^{*}}\right)^{2}}+\frac{\lambda^{2 \underline{n}_{e}^{*}}\left(\lambda^{\bar{n}_{e}^{*}}+1\right)^{2}}{\left(1-\lambda^{\bar{n}_{e}^{*}}\right)\left(\lambda \underline{n}_{e}^{*}+1\right)^{3}}\right)\right)
\end{aligned}
$$

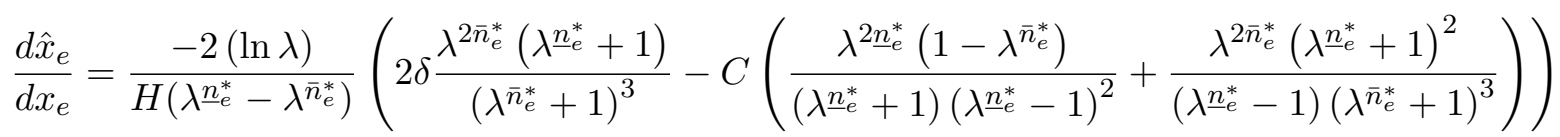

From the proof of Lemma 1, we know that for $x_{e}=1 / 2, d \tilde{x}_{e} / d x_{e}=d \hat{x}_{e} / d x_{e}>0$. Hence, for $x_{e}=1 / 2, \frac{d \tilde{x}_{e} / d x_{e}}{d \hat{x}_{e} / d x_{e}}=1$. More generally, for any $x_{e}$ :

$$
\frac{d \tilde{x}_{e}}{d x_{e}}-\frac{d \hat{x}_{e}}{d x_{e}}=\left(1-\lambda^{n_{e}^{*}} \lambda^{\bar{n}_{e}^{*}}\right)\left(\lambda^{n_{e}^{*}}-\lambda^{\bar{n}_{e}^{*}}\right) \cdot \alpha \cdot \beta
$$

where

$$
\alpha=2 \delta \frac{\left(4 \lambda^{\bar{n}_{e}^{*}} \lambda \underline{n}_{e}^{*}+\left(\lambda^{\underline{n}_{e}^{*}}+\lambda^{\bar{n}_{e}^{*}}\right)\left(1+\lambda \underline{\underline{n}}_{e}^{*} \lambda^{\bar{n}_{e}^{*}}\right)\right)}{\left(\lambda \underline{\underline{n}}_{e}^{*}+1\right)^{3}\left(\lambda^{\bar{n}_{e}^{*}}+1\right)^{3}}
$$

and

$$
\beta=4 C \frac{\left(\frac{\lambda^{2 \bar{n}_{e}^{*}}}{\left(\lambda^{\bar{n}_{e}^{*}}+1\right)^{2}\left(1-\lambda^{2 \bar{n}_{e}^{*}}\right)}+\frac{\lambda^{2 \underline{n}_{e}^{*}}}{\left(\lambda_{\underline{\underline{n}}}^{*}+1\right)^{2}\left(\lambda^{2 \underline{n}_{e}^{*}}-1\right)}\right)}{\left(\lambda^{\underline{n}_{e}^{*}}-1\right)\left(1-\lambda^{\bar{n}_{e}^{*}}\right)}
$$

where $\alpha$ and $\beta$ are always positive. Hence given (20):

$$
\frac{d \tilde{x}_{e} / d x_{e}}{d \hat{x}_{e} / d x_{e}}\left\{\begin{array}{l}
>1 \text { for } x_{e}<\frac{1}{2} \\
=1 \text { for } x_{e}=\frac{1}{2} \\
<1 \text { for } x_{e}>\frac{1}{2}
\end{array}\right.
$$

In other words, for $x_{e}>\frac{1}{2}$ an increase in $x_{e}$ increases $\hat{x}_{e}$ more than $\tilde{x}_{e}$ (and viceversa for $x_{e}<\frac{1}{2}$ ). Then, it is immediate to verify that when the distribution of citizens' idiosyncratic preferences is such that Condition A is verified, then $x_{e}=\frac{1}{2}$ is the unique stationary point and the global maximum.

Now suppose $F(x)$ is such that Condition B is verified. For $x_{e_{R}}>\frac{1}{2}$ to be a stationary point it must be the case that $f\left(\hat{x}_{e_{R}}\left(\bar{n}_{e}^{*}, \underline{n}_{e}^{*}\right)\right)<f\left(\tilde{x}_{e_{R}}\left(\bar{n}_{e}^{*}, \underline{n}_{e}^{*}\right)\right)$. Moreover, from Lemma 1 and (29) we know 
that for $x_{e_{R}}>1 / 2$, then $\tilde{x}_{e_{R}}\left(\bar{n}_{e}^{*}, \underline{n}_{e}^{*}\right)>1-\hat{x}_{e_{R}}\left(\bar{n}_{e}^{*}, \underline{n}_{e}^{*}\right)$. Then, $x_{e}=\frac{1}{2}$ cannot be a global maximum since $\left.\frac{d f(x)}{d x}\right|_{x=1 / 2}>0$ and $\left.\frac{d \tilde{x}_{e}}{d x_{e}}\right|_{x=1 / 2}=\left.\frac{d \hat{x}_{e}}{d x_{e}}\right|_{x=1 / 2}$. Thus the stationary point $x_{e_{R}}^{m o n}>\frac{1}{2}$ such that (28) is satisfied will be a global maximum on $\left(\frac{1}{2}, 1\right)$. Then by the symmetry of $f$, choosing an editor with symmetric preferences will also be profit-maximizing. That is, we have two global maxima in this case $x_{e_{R}}^{m o n}$ and $x_{e_{L}}^{m o n}=1-x_{e_{R}}^{m o n}$. Indeed, since the distribution function $f$ is symmetric around $\frac{1}{2}$, so it must be the demand function. To sum up, if $F(x)$ is such that Condition A holds the global maximum is always at $x_{e}=\frac{1}{2}$. Instead, if $F(x)$ is such that Condition B holds, there are two symmetric global maxima such that $x_{e_{R}}=1-x_{e_{L}}>1 / 2$. The last part of the proposition follows immediately from Lemma 1 Q.E.D.

\section{Proof of Proposition 3}

Let's start with the case where Condition A holds. We show that in this case the unique equilibrium is such that $x_{e}^{1}=x_{e}^{2}=\frac{1}{2}$. Suppose that media outlet 1 deviates by choosing $x_{e}^{1}>x_{e}^{2}=\frac{1}{2}$. If media outlet one deviates, the indifferent viewer, i.e., the viewer who will be indifferent between watching media outlet 1 and media outlet 2 is the one having preferences $x_{I}$ such that $U_{I}\left(W_{1}\right)=U_{I}\left(W_{2}\right)$. That is:

$$
x_{I}\left(\bar{n}_{e_{1}}^{*}, \underline{n}_{e_{1}}^{*}, \bar{n}_{e_{2}}^{*}\right)=\frac{1}{2}+\frac{\delta}{\left(\lambda^{\underline{n}_{e_{1}}^{*}} \lambda^{\bar{n}_{e_{1}}^{*}}-1\right)}\left(\frac{\left(1-\lambda^{\bar{n}_{e_{2}}^{*}}\right)\left(\lambda^{\underline{n}_{e_{1}}^{*}}-\lambda^{\bar{n}_{e_{1}}^{*}}\right)}{\left(\lambda^{\bar{n}_{e_{2}}^{*}}+1\right)}-\left(\lambda^{\underline{n}_{e_{1}}^{*}}-1\right)\left(1-\lambda^{\bar{n}_{e_{1}}^{*}}\right)\right)
$$

where since $x_{e}^{2}=\frac{1}{2}$, then $\bar{n}_{e_{2}}^{*}=-\underline{n}_{e_{2}}^{*}$. The no-deviation condition for media outlet 1 requires that $\nexists x_{e}>\frac{1}{2}$ such that the demand if deviating is higher than the demand if not deviating. Specifically, the demand that media outlet 1 faces when not deviating is:

$$
D^{N D e v}\left(x_{e}^{1}\right)=D^{N D e v}\left(x_{e}^{2}\right)=\frac{1}{2}\left[F\left(\left.\tilde{x}_{e}\right|_{x_{e}=\frac{1}{2}}\right)-F\left(\left.\hat{x}\right|_{x_{e}=\frac{1}{2}}\right)\right]=\left[F\left(\left.\tilde{x}_{e}\right|_{x_{e}=\frac{1}{2}}\right)-F\left(\frac{1}{2}\right)\right]
$$

Instead the demand that media outlet 1 faces if it deviates is:

$$
D^{D e v}\left(x_{e}^{1}\right)=\left[F\left(\left.\tilde{x}_{e}\right|_{x_{e}^{1}}\right)-F\left(\max \left\{\hat{x}_{e_{1}} ; x_{I}\left(x_{e_{1}}\right)\right\}\right)\right]
$$

Notice that for any non-uniform distribution satisfying Condition A the mass of citizens is strictly decreasing moving away from the mean of the distribution at $1 / 2$. Hence it is enough to show that this no-deviation condition holds even in the case where citizens' preferences are uniformly distributed in $[0,1] .^{42}$ In the case of a uniform distribution, the following represents a sufficient no-deviation condition:

$$
x_{I}\left(\bar{n}_{e_{1}}^{*}, \underline{n}_{e_{1}}^{*}, \bar{n}_{e_{2}}^{*}\right)-\frac{1}{2}>\left.\tilde{x}_{e}\right|_{x_{e}^{1}}-\left.\tilde{x}_{e}\right|_{x_{e}=\frac{1}{2}}
$$

hence media outlet 1 would not deviate if and only if:

$$
C>C^{T H R}=\delta \frac{\left(\lambda^{2 \underline{n}_{e_{1}}^{*}}-1\right)\left(1-\lambda^{\bar{n}_{e_{1}}^{*}}\right)}{\left(\lambda^{\underline{n}_{e_{1}}^{*}} \lambda^{\bar{n}_{e_{1}}^{*}}-1\right)^{2}}\left(\frac{\left(\lambda^{\underline{n}_{e_{1}}^{*}}-\lambda^{\bar{n}_{e_{1}}^{*}}\right)}{\left(\lambda^{\underline{\underline{n}}_{e_{1}}^{*}}+1\right)}-\frac{\left(1-\lambda^{\bar{n}_{e_{2}}^{*}}\right)}{\left(\lambda^{\bar{n}_{e_{2}}^{*}}+1\right)}\left(\lambda^{\bar{n}_{e_{1}}^{*}}+1\right)\right)
$$

where $C^{T H R}>0$ if and only if

$$
\frac{\left(\lambda^{\underline{n}_{e_{1}}^{*}}-\lambda^{\bar{n}_{e_{1}}^{*}}\right)}{\left(\lambda^{\underline{n}_{e_{1}}^{*}}+1\right)\left(\lambda^{\bar{n}_{e_{1}}^{*}}+1\right)}>\frac{\left(1-\lambda^{\bar{n}_{e_{2}}^{*}}\right)}{\left(\lambda^{\bar{n}_{e_{2}}^{*}}+1\right)}
$$

\footnotetext{
${ }^{42}$ Notice also that, as stated in section 2.1 the analysis focuses on symmetric distributions.
} 
Let $A=\frac{\left(\lambda^{\underline{n}_{e}^{*}}-\lambda^{\bar{n}_{e_{1}}^{*}}\right)}{\left(\lambda^{n_{e_{1}^{*}}^{*}}+1\right)\left(\lambda^{\bar{n}_{e_{1}}^{*}}+1\right)}$. For $x_{e}>\frac{1}{2}, \frac{d A}{d x_{e}}<0$ which implies that:

$$
\frac{\left(\lambda^{\underline{n}_{e_{1}}^{*}}-\lambda^{\bar{n}_{e_{1}}^{*}}\right)}{\left(\lambda^{\underline{n}_{e_{1}}^{*}}+1\right)\left(\lambda^{\bar{n}_{e_{1}}^{*}}+1\right)}<\left.\frac{\left(\lambda^{\underline{n}_{e_{1}}^{*}}-\lambda^{\bar{n}_{e_{1}}^{*}}\right)}{\left(\lambda^{\underline{n}_{e_{1}}^{*}}+1\right)\left(\lambda^{\bar{n}_{e_{1}}^{*}}+1\right)}\right|_{x_{e}=\frac{1}{2}}=\frac{\left(1-\lambda^{\bar{n}_{e_{2}}^{*}}\right)}{\left(\lambda^{\bar{n}_{e_{2}}^{*}}+1\right)}
$$

hence $C^{T H R}<0$. Therefore, in a duopoly when the distribution of citizens' idiosyncratic preferences is such that Condition A holds (and where citizens watch at most one media report), there will never be an incentive to deviate from the equilibrium at $x_{e}^{1}=1-x_{e}^{2}=\frac{1}{2}$. Moreover, notice that this is the unique Nash equilibrium. If the two media outlets were to choose ideological editors, then each of them would clearly have an incentive to deviate by choosing a moderate one.

Let's now analyze the case where Condition B holds. First of all, in order to ensure that there is someone willing to watch media 1 the following condition must be satisfied

$$
x_{I}\left(\bar{n}_{e_{1}}^{*}, \underline{n}_{e_{1}}^{*}, \bar{n}_{e_{2}}^{*}\right)<\tilde{x}_{e}\left(x_{e}^{1}\right)
$$

that is:

$$
C<\bar{C}=2 \delta \frac{\left(1-\lambda^{\bar{n}_{e_{1}}^{*}}\right)}{\left(\lambda^{\bar{n}_{e_{2}}^{*}}+1\right)}
$$

where clearly $\bar{C}>0 .{ }^{43}$ Let's now analyze the no-deviation condition for $C<\bar{C}$. Consider (30) and (31) and let $C^{\text {Duop }}=C^{\text {Duop }}\left(x_{e_{1}}\right)$ be the highest opportunity cost such that for $x_{e_{1}} \in\left(\frac{1}{2}, 1\right)$ the following condition holds (i.e., $C^{\text {Duop }}$ being the opportunity cost associated with the most profitable deviation from $\left.x_{e_{1}}=1 / 2\right):{ }^{44}$

$$
\frac{F\left(\max \left\{\hat{x}_{e_{1}} ; x_{I}\left(x_{e_{1}}\right)\right\}\right)-\frac{1}{2}}{F\left(\tilde{x}_{e_{1}}\right)-F\left(\left.\tilde{x}_{e}\right|_{x_{e}=\frac{1}{2}}\right)} \geq 0
$$

now denote $C^{D e v}=\min \left\{\bar{C}, C^{D u o p}\right\}$, then for $C \in\left(0, C^{D e v}\right)$ media outlet 1 will have an incentive to deviate by choosing an ideological editor. ${ }^{45}$ Hence, in such case there is no equilibrium where both media outlets choose a moderate editor. ${ }^{46}$ Let's now show that it can never exist an equilibrium with $x_{e_{1}}=x_{e_{2}} \neq \frac{1}{2}$. Suppose the two media outlets choose the same type of ideological editors (e.g., $x_{e_{1}}=x_{e_{2}}>\frac{1}{2}$ ). By doing so their demand would be

$$
D^{1}\left(x_{e_{1}}=x_{e_{2}}\right)=D^{2}\left(x_{e_{1}}=x_{e_{2}}\right)=\frac{F\left(\tilde{x}_{e_{1}}\right)-F\left(\hat{x}_{e_{1}}\right)}{2}
$$

while if media outlet 2 chooses an editor with preferences $x_{e_{2}}=1-x_{e_{1}}$ its demand would be:

$$
D^{2}\left(x_{e_{2}}=1-x_{e_{1}}\right)=\min \left\{F\left(\tilde{x}_{e_{2}}\right) ; \frac{1}{2}\right\}-F\left(\hat{x}_{e_{2}}\right)
$$

where by symmetry $\hat{x}_{e_{2}}=1-\tilde{x}_{e_{1}}$, which implies that $F\left(\hat{x}_{e_{2}}\right)=1-F\left(\tilde{x}_{e_{1}}\right)$. Thus, a necessary ${ }^{43}$ Notice also that $x_{I}\left(\bar{n}_{e_{1}}^{*}, \underline{n}_{e_{1}}^{*}, \bar{n}_{e_{2}}^{*}\right)<\left.\tilde{x}_{e}\right|_{x_{e}=\frac{1}{2}}$ if and only if $C<\check{C} \equiv 2 \delta \frac{\left(1-\lambda^{\bar{n}_{e_{1}}^{*}}\right)}{\lambda^{\bar{n}_{e_{2}}^{*}}+1} \frac{\lambda_{\underline{n}_{e^{*}}^{*}}^{n_{n^{*}}^{*}} \lambda^{\bar{n}_{e_{1}}^{*}} \lambda^{\bar{n}_{e_{1}}^{*}}-1}{\bar{n}_{e_{1}}^{*}}$ where $\check{C}>0$ since $\left|\underline{n}_{e_{1}}^{*}\right|>\bar{n}_{e_{2}}^{*}$.

${ }^{44}$ Since $f(x)$ is assumed to be symmetric with respect to $1 / 2$, the mean and the median will always be at $1 / 2$. Hence $F(1 / 2)=1 / 2$.

${ }^{45}$ Clearly, if $C^{D e v}<0$, firm 1 will never have an incentive to deviate. Indeed, as shown in the previous case where $\left(\right.$ Condition $A$ ) holds, when $F$ is a uniform c.d.f. $C^{D e v}=C^{T H R}<0$.

${ }^{46} C^{D e v}$ is always lower than $C^{\max }$ since for $C=C^{\max }$ only citizens with $x_{e}=\frac{1}{2}$ watch news reports and thus firm 1 would never have an incentive to deviate. 
condition for media outlet 2 not be willing to deviate is $1-F\left(\hat{x}_{e_{1}}\right)>F\left(\tilde{x}_{e_{1}}\right)$. However, since $x_{e_{1}}>\frac{1}{2}$, then $\tilde{x}_{e_{1}}>1-\hat{x}_{e_{1}}$ and given Condition B this condition cannot hold. An analogous proof applies for $x_{e_{1}}=x_{e_{2}}<\frac{1}{2}$. Hence, for $C \in\left(0, C^{D e v}\right)$ the only possible Nash Equilibrium must be such that $x_{e_{1}}=1-x_{e_{2}} \neq 1 / 2$. Let's show that this is indeed an equilibrium. ${ }^{47}$ Suppose that $x_{e_{1}}=1-x_{e_{2}}>\frac{1}{2}$, then there are two possible cases. In the first one, $\forall x_{e_{1}}=1-x_{e_{2}} \in\left(\frac{1}{2}, x_{e_{R}}^{\max }\right)$ it is always the case that: ${ }^{48}$

$$
\left.\frac{d F\left(\max \left\{\hat{x}_{e_{1}} ; x_{I}\left(x_{e_{1}}\right)\right\}\right)}{d x_{e_{1}}}\right|_{x_{e_{1}}=1-x_{e_{2}}}<\left.\frac{d F\left(\tilde{x}_{e_{1}}\right)}{d x_{e_{1}}}\right|_{x_{e_{1}}=1-x_{e_{2}}}
$$

where for $x_{e_{1}}=1-x_{e_{2}}, x_{I}\left(x_{e_{1}}\right)$ is always $1 / 2$. Hence in this case $x_{e_{1}}=1-x_{e_{2}}=x_{e_{R}}^{\max }$ is a Nash equilibrium. Indeed, by Lemma $1, \tilde{x}_{e_{1}}$ is increasing in $x_{e_{1}}$ if and only if $x_{e_{1}}<x_{e_{R}}^{\max }$. Hence, $\left.\frac{d F\left(\tilde{x}_{e_{1}}\right)}{d x_{e_{1}}}\right|_{x_{e_{1}=1-x_{e_{2}}}}>0$ if and only if $x_{e_{1}}<x_{e_{R}}^{\max }$. On the other hand, since by Lemma 1, for $x_{e_{1}}>1 / 2, \hat{x}_{e_{1}}$ is always increasing in $x_{e_{1}}$ and $x_{I}$ is increasing in $x_{e_{1}}$ when $x_{e_{1}}=1-x_{e_{2}}$ (i.e., for $\left.x_{I}=1 / 2\right)$. Thus given Condition B it is always the case that $\left.\frac{d F\left(\max \left\{\hat{x}_{e_{1}} ; x_{I}\left(x_{e_{1}}\right)\right\}\right)}{d x_{e_{1}}}\right|_{x_{e_{1}}=1-x_{e_{2}}}>0$. Thus, none of the two media outlet would have an incentive to deviate from $x_{e_{1}}=1-x_{e_{2}}=x_{e_{R}}$ by choosing a more leftist or more rightist editor. In the second case, $\exists x_{e_{1}} \in\left(\frac{1}{2}, x_{e_{R}}^{\max }\right)$ such that (35) is not verified. Hence, since by construction of $C^{D e v}$, for $C<C^{D e v}$ :

$$
\left.\frac{d F\left(\max \left\{\hat{x}_{e_{1}} ; x_{I}\left(x_{e_{1}}\right)\right\}\right)}{d x_{e_{1}}}\right|_{x_{e_{1}}=1-x_{e_{2}}=1 / 2}<\left.\frac{d F\left(\tilde{x}_{e_{1}}\right)}{d x_{e_{1}}}\right|_{x_{e_{1}}=1-x_{e_{2}}=1 / 2}
$$

then it will always exist a $x_{e_{1}} \in\left(\frac{1}{2}, x_{e_{R}}^{\max }\right)$ such that:

$$
\left.\frac{d F\left(\max \left\{\hat{x}_{e_{1}} ; x_{I}\left(x_{e_{1}}\right)\right\}\right)}{d x_{e_{1}}}\right|_{x_{e_{1}}=1-x_{e_{2}}}=\left.\frac{d F\left(\tilde{x}_{e_{1}}\right)}{d x_{e_{1}}}\right|_{x_{e_{1}}=1-x_{e_{2}}}
$$

that is, $x_{e_{1}}=1-x_{e_{2}} \in\left(\frac{1}{2}, x_{e_{R}}^{\max }\right)$ is a Nash equilibrium. Finally, we need to show that a lower $C$ is associated with a Nash equilibrium where the difference between the idiosyncratic preferences of the editors chosen by each media outlet, i.e., $\left|x_{e_{1}}-x_{e_{2}}\right|$, is higher. First of all by Lemma 1, a lower $C$ corresponds to a higher $x_{e_{R}}^{\max }$ and a lower $x_{e_{L}}^{\min }$. Moreover, since as $C$ decreases $\frac{d \tilde{x}_{e}\left(\bar{n}_{e_{1}}^{*}, \underline{n}_{e_{1}}^{*}\right)}{d x_{e}}$ increases, hence $\left.\frac{d F\left(\tilde{x}_{e_{1}}\right)}{d x_{e_{1}}}\right|_{x_{e_{1}}=1-x_{e_{2}}}$ increases as well. Thus since the RHS of (36), the LHS must increase as well, which, in turn implies that $x_{e_{1}}$ must be higher (similarly, $x_{e_{2}}$ will be lower). That is, a lower $C$ is associated with an equilibrium where the two media outlets choose less moderate editors. Q.E.D.

\section{Proof of Proposition 4}

We have to analyze the no-deviation condition with $K$ media outlets. Let $\bar{n}_{e}^{*}=-\underline{n}_{e}^{*}$ be the stopping thresholds chosen by a moderate editor. The demand media outlet 1 faces if it chooses a moderate editor as all the other media outlets is $\forall j \in\{2,3, \ldots ., K\}$ :

$$
D^{N D e v}\left(x_{e}^{1}\right)=D^{N D e v}\left(x_{e}^{j}\right)=\frac{1}{K}\left[F\left(\left.\tilde{x}\right|_{x_{e}=\frac{1}{2}}\right)-F\left(\left.\hat{x}\right|_{x_{e}=\frac{1}{2}}\right)\right]=\frac{2}{K}\left[F\left(\left.\tilde{x}\right|_{x_{e}=\frac{1}{2}}\right)-F\left(\frac{1}{2}\right)\right]
$$

Instead the demand that media outlet 1 faces if it deviates from such position is:

$$
D^{D e v}\left(x_{e}^{1}\right)=\left[F\left(\left.\tilde{x}\right|_{x_{e}^{1}}\right)-F\left(\max \left\{\hat{x}_{e_{1}} ; x_{I}\left(x_{e_{1}}\right)\right\}\right)\right]
$$

\footnotetext{
${ }^{47}$ Obviously, for $C \in\left(0, C^{D e v}\right)$ there are always two symmetric Nash Equilibria, i.e., $x_{e_{1}}=1-x_{e_{2}}<\frac{1}{2}$ and $x_{e_{1}}=1-x_{e_{2}}>\frac{1}{2}$.

${ }^{48}$ Symmetric conditions apply for media outlet 2 .
} 
Hence given a uniform distribution, media outlet 1 will prefer not to choose a moderate editor if and only if:

$$
K>K^{*}=\frac{2\left[\left.\tilde{x}\right|_{x_{e}=\frac{1}{2}}-\frac{1}{2}\right]}{\left.\tilde{x}\right|_{x_{e}^{1}}-\max \left\{\hat{x}_{e_{1}} ; x_{I}\left(x_{e_{1}}\right)\right\}}
$$

where we know from the proof of Proposition 3, that $K^{*}>2$. Moreover, the game satisfies the properties of Theorem 4 in Dasgupta and Maskin (1986) for the existence of an equilibrium in a product competition game. Hence, the $K^{*}$ media outlets game possesses a symmetric mixedstrategy Nash equilibrium. Moreover it is always the case that $\frac{d K^{*}}{d C}>0$ since $\hat{x}_{e_{1}}$ is increasing in $C$ and $d \tilde{x}_{e} / d x_{e}$ is decreasing in $C$. Q.E.D. 
1. L. Colombo, H. Dawid, Strategic Location Choice under Dynamic Oligopolistic Competition and Spillovers, Novembre 2013.

2. M. Bordignon, M. Gamalerio, G. Turati, Decentralization, Vertical Fiscal Imbalance, and Political Selection, Novembre 2013.

3. M. Guerini, Is the Friedman Rule Stabilizing? Some Unpleasant Results in a Heterogeneous Expectations Framework, Novembre 2013.

4. E. Brenna, C. Di Novi, Is caring for elderly parents detrimental to women's mental health? The influence of the European North-South gradient, Novembre 2013.

5. F. Sobbrio, Citizen-Editors' Endogenous Information Acquisition and News Accuracy, Novembre 2013. 